\title{
SOLUTION OF THE TRUNCATED PARABOLIC MOMENT PROBLEM
}

\author{
RAÚL E. CURTO AND LAWRENCE A. FIALKOW
}

\begin{abstract}
Given real numbers $\beta \equiv \beta^{(2 n)}=\left\{\beta_{i j}\right\}_{i, j \geq 0, i+j \leq 2 n}$, with $\gamma_{00}>0$, the truncated parabolic moment problem for $\beta$ entails finding necessary and sufficient conditions for the existence of a positive Borel measure $\mu$, supported in the parabola $p(x, y)=0$, such that $\beta_{i j}=\int y^{i} x^{j} d \mu(0 \leq i+j \leq 2 n)$. We prove that $\beta$ admits a representing measure $\mu$ (as above) if and only if the asociated moment matrix $\mathcal{M}(n)(\beta)$ is positive semidefinite, recursively generated and has a column relation $p(X, Y)=$ 0 , and the algebraic variety $\mathcal{V}(\beta)$ associated to $\beta$ satisfies card $\mathcal{V}(\beta) \geq \operatorname{rank} \mathcal{M}(n)(\beta)$. In this case, $\beta$ admits a $\operatorname{rank} \mathcal{M}(n)$-atomic (minimal) representing measure.
\end{abstract}

\section{INTRODUCTION}

Given complex numbers $\gamma \equiv \gamma^{(2 n)}: \gamma_{00}, \gamma_{01}, \gamma_{10}, \ldots, \gamma_{0,2 n}, \ldots, \gamma_{2 n, 0}$, with $\gamma_{i j}=\bar{\gamma}_{j i}$, the Truncated Complex Moment Problem (TCMP) for $\gamma$ entails finding conditions for the existence of a positive Borel measure $\mu$, supported in the complex plane $\mathbb{C}$, such that

$$
\gamma_{i j}=\int \bar{z}^{i} z^{j} d \mu \quad(0 \leq i+j \leq 2 n) .
$$

In [CuFi1], [CuFi3] and [CuFi4], we initiated a study of TCMP based on positivity and extension properties of the moment matrix $M(n) \equiv M(n)(\gamma)$ associated to $\gamma \equiv \gamma^{(2 n)}$. As we describe below, corresponding to each polynomial $p(z, \bar{z})$ with $\operatorname{deg} p \leq n$, there is an element $p(Z, \bar{Z})$ in the column space of $M(n)$; under this correspondence, each column dependence relation in $M(n)$ can be expressed as $p(Z, \bar{Z})=0$ for a suitable polynomial $p$. We define the algebraic variety of $\gamma$ by $\mathcal{V}\left(\gamma^{(2 n)}\right):=\bigcap_{p(Z, \bar{Z})=0, \operatorname{deg} p \leq n} \mathcal{Z}(p)$, where $\mathcal{Z}(p)$ denotes the zero set of $p$. We say that $M(n)$ is recursively generated if whenever $p(Z, \bar{Z})=0$ and $\operatorname{deg} p q \leq n$, then $(p q)(Z, \bar{Z})=0$. In order for $\gamma^{(2 n)}$ to have a representing measure it is necessary that $M(n)$ be positive, recursively generated, and satisfy rank $M(n) \leq \operatorname{card} \mathcal{V}\left(\gamma^{(2 n)}\right)$. Remarkably, to date there are no known examples of singular $M(n)$ for which these necessary conditions are not also sufficient. In the present paper, we establish that these necessary conditions are indeed sufficient for truncated moment problems subordinate to parabolic curves in the plane.

In $[\mathrm{CuFi}]$ we characterized existence and uniqueness of representing measures in the singular quartic moment problem, the case of TCMP with $n=2$ for which $M(2)$ is singular. The singularity of $M(2)$ forces nontrivial dependence relations in its columns which correspond to second-degree algebraic relations in the support of any representing measure. Indeed, a representing measure for

1991 Mathematics Subject Classification. Primary 47A57, 44A60, 42A70, 30A05; Secondary 15A57, 15-04, 47N40, 47A20.

Key words and phrases. Parabolic moment problem, moment matrix extension, flat extensions of positive matrices

The first-named author's research was partially supported by NSF Research Grant DMS-0099357. The secondnamed author's research was partially supported by NSF Research Grant DMS-0201430. The second-named author was also partially supported by the State University of New York at New Paltz Research and Creative Projects Award Program. 
$\gamma^{(4)}$ must be supported in the algebraic variety $\mathcal{V}\left(\gamma^{(4)}\right)$, defined (as above) as the intersection of the zero sets of all second-degree polynomials corresponding to column dependence relations in $M(2)$. In the present note we generalize some of the results in [CuFi6] so as to characterize the existence and uniqueness of representing measures for any $M(n)(\gamma)(n \geq 2)$ which has a parabolic-type dependence relation in its columns.

Recall from [CuFi1, Corollary 3.7] that if $\gamma^{(2 n)}$ admits a representing measure $\mu$, then $M(n)$ is positive semidefinite $(M(n) \geq 0)$, recursively generated, and card $\operatorname{supp} \mu \geq \operatorname{rank} M(n)$. The main result of [CuFi1] shows that $\gamma^{(2 n)}$ has a $\operatorname{rank} M(n)$-atomic (minimal) representing measure if and only if $M(n) \geq 0$ admits a flat extension, i.e., an extension to a moment matrix $M(n+1)$ satisfying $\operatorname{rank} M(n+1)=\operatorname{rank} M(n)$ [CuFi1, Theorem 5.13] . In particular, if $M(n)$ is flat, i.e., $\operatorname{rank} M(n-1)=\operatorname{rank} M(n)$, then $M(n)$ admits a flat extension [CuFi1, Theorem 5.4]. Let us denote the successive columns of $M(n)$ lexicographically, by $1, Z, \bar{Z}, \ldots, Z^{n}, \bar{Z} Z^{n-1}, \ldots, \bar{Z}^{n}$. Results of [CuFi2] imply that for $n \geq 2$, if $M(n) \geq 0$ is recursively generated and $\left\{1, Z, \bar{Z}, Z^{2}\right\}$ is dependent in $\mathcal{C}_{M(n)}$ (the column space of $M(n)$ ), then $M(n)$ admits a flat extension $M(n+1)$ (and a corresponding rank $M(n)$-atomic (minimal) representing measure) (cf. Theorems 1.2 and 1.4 below). Other concrete sufficient conditions for flat extensions $M(n+1)$ were obtained in [CuFi3] and [CuFi6], but a complete solution to the Flat Extension Problem remains unknown.

In our analysis of the quartic moment problem in [CuFi6], to study column relations in $M(2)(\gamma)$, we reduced the problem to four cases, naturally associated to the real conics $x^{2}+y^{2}=1, y=x^{2}$, $x y=1$ and $x y=0$. To facilitate this approach using real conics, we replaced $M(2)(\gamma)$ with an equivalent real moment matrix $\mathcal{M}(2)(\beta)$. In the present note we use a similar approach. We replace $M(n)(\gamma)$ with an associated real moment matrix $\mathcal{M}(n) \equiv \mathcal{M}(n)(\beta)$, where $\beta \equiv \beta^{(2 n)}$ is a real sequence $\beta_{i j}(0 \leq i+j \leq 2 n)$ naturally corresponding to $\gamma^{(2 n)}$ (cf. [CuFi6]). The columns of $\mathcal{M}(n)$ are indexed by $1, X, Y, X^{2}, Y X, Y^{2}, \ldots, X^{n}, \ldots, Y^{n}$, and we focus on a column relation of the form $Y=X^{2}$; indeed, the general truncated moment problem for measures supported in a parabola can be reduced to this case [CuFi6, paragraph following Lemma 5.1]. Our main result (Theorem 1.4) shows that $\beta^{(2 n)}$ admits a representing measure supported in $y=x^{2}$ if and only if $\mathcal{M}(n)$ is positive, recursively generated, has a column relation $Y=X^{2}$, and satisfies the condition $\operatorname{card} \mathcal{V}(\beta) \geq \operatorname{rank} \mathcal{M}(n)(\beta)$. In proving Theorem 1.4, we occasionally refer to results for TCMP and $M(n)(\gamma)$. Due to the equivalence of $M(n)(\gamma)$ and $\mathcal{M}(n)(\beta)$, the results for $M(n)(\gamma)$ have exact analogues for $\mathcal{M}(n)(\beta)$. In the sequel we repeatedly exploit this equivalence, often without further reference.

Part of the motivation for the present study comes from the Full Moment Problem. For $\beta \equiv$ $\beta^{(\infty)} \equiv\left\{\beta_{i j}\right\}_{i, j \geq 0}$, let $\mathcal{M} \equiv \mathcal{M}(\infty)(\beta)$, and for a polynomial $p \in \mathbb{R}[x, y]$, let $p(X, Y)$ denote the corresponding element of $\mathcal{C}_{\mathcal{M}}$, the column space of $\mathcal{M}$. In order to have a representing measure for $\beta$ supported in $\mathcal{Z}(p) \equiv\{(x, y): p(x, y)=0\}$, it is necessary that $\mathcal{M} \geq 0$ and $p(X, Y)=0$. Following J. Stochel [Sto1], we say that $p$ is of type $A$ if the conditions $\mathcal{M} \geq 0$ and $p(X, Y)=0$ imply that $\beta^{(\infty)}$ has a representing measure (necessarily supported in $\mathcal{Z}(p)$ ). In [Sto1, Theorem 5.4], Stochel proved that if $\operatorname{deg} p \leq 2$, then $p$ is of type A, and Stochel and Szafraniec [StSz1, Theorem 4] showed that there exist type A polynomials of arbitrarily large degree. By contrast, Stochel [Sto1] proved that there exist polynomials of degree 3 (e.g., $y\left(y-x^{2}\right)$ ) that are not of type A. The present work is part of an attempt to develop an analogue of type A for the truncated moment problem. In particular, 
following [Fia3], we are studying whether every polynomial $p$ in $x$ and $y$ of degree at most $n$ satisfies $\beta^{(2 n)}$ has a representing measure supported in $\mathcal{Z}(p)$ if and only if

$$
\begin{aligned}
& \mathcal{M}(n) \geq 0, \mathcal{M}(n) \text { is recursively generated, } \\
& \qquad p(X, Y)=0 \text { and } \operatorname{rank} \mathcal{M}(n) \leq \operatorname{card} \mathcal{V}\left(\beta^{(2 n)}\right) . \quad\left(A_{n}^{\prime}\right)
\end{aligned}
$$

(Of course, $\left(A_{n}^{\prime}\right)$ can also be formulated for $p(z, \bar{z}), \gamma^{(2 n)}$ and $M(n)(\gamma)$.) Note that the properties in $\left(A_{n}^{\prime}\right)$ can be verified using only elementary linear algebra and tools for solving polynomial equations. Thus, if a polynomial $p$ satisfies $\left(A_{n}^{\prime}\right)$, then any $\mathcal{M}(n)$ moment problem subordinate to $p(x, y)=0$ can be resolved by entirely elementary methods.

In the present paper, we prove that any parabolic polynomial $p$ satisfies $\left(A_{n}^{\prime}\right)$ for $n \geq 2$ (cf. Theorem 1.4), and we use this result to recover Stochel's degree 2 theorem in the case of parabolas (cf. Theorem 2.3). In the forthcoming paper [CuFi8] we establish $\left(A_{n}^{\prime}\right)$ for hyperbolic polynomials. These results, together with those in $[\mathrm{CuFi} 2]$ and $[\mathrm{CuFi} 6]$, show that any polynomial of degree at most 2 satisfies $\left(A_{n}^{\prime}\right)$ for $n \geq 2$, which implies a new proof of [Sto1, Theorem 5.4] (cf. [CuFi8]). To motivate Theorem 1.4, we next review some of the results of [CuFi2], [CuFi6] and [Fia3]. For a truncated moment problem whose associated moment matrix $M(n)$ is positive, recursively generated and satisfies an analytic linear column relation $Z=c 1$, it is easy to see that $\mu:=\delta_{c}$ is the unique representing measure. For complex lines, we have the following result.

Theorem 1.1. ([CuFi2, Theorem 2.1]) If $M(n)$ is positive, recursively generated, and satisfies $\bar{Z}=$ $a 1+b Z(b \neq 0)$ in $\mathcal{C}_{M(n)}$, then $\gamma^{(2 n)}$ admits infinitely many rank $M(n)$-atomic (minimal) representing measures, each supported in the line $\bar{z}=a+b z$.

In [CuFi2] we established the existence of a rank $M(n)$-atomic representing measure whenever $M(n)$ is positive, recursively generated and has a column relation $Z^{k}=q(Z, \bar{Z})$, where $\operatorname{deg} q<$ $k \leq\left[\frac{n}{2}\right]+1$. (This is actually the unique representing measure for $\gamma^{(2 n)}$ [CuFi7, Proposition 4.2].) In particular, [CuFi2, Theorem 3.1] implies that if $M(n)$ is positive, recursively generated, and $Z^{2}=a 1+b Z+c \bar{Z}$, then $\gamma^{(2 n)}$ admits a unique $\operatorname{rank} M(n)$-atomic representing measure.

In $[\mathrm{CuFi} 6]$ we treated the case when $\{1, Z, \bar{Z}\}$ is independent and $\bar{Z} Z=a 1+b Z+c \bar{Z}$; this case encompasses measures supported in a circle.

Theorem 1.2. ([CuFi6, Theorem 2.1]) If $M(n)$ is positive, recursively generated, $\{1, Z, \bar{Z}\}$ is independent in $\mathcal{C}_{M(n)}$, and $\bar{Z} Z=a 1+b Z+c \bar{Z}$, then $\gamma^{(2 n)}$ admits a rank $M(n)$-atomic representing measure, necessarily supported in the circle $|z-\bar{b}|^{2}=a+|b|^{2}(>0)$. Moreover, $\operatorname{rank} M(n) \leq 2 n+1$, and if $\operatorname{rank} M(n) \leq 2 n$, then $\gamma^{(2 n)}$ has a unique representing measure. If $\operatorname{rank} M(n)=2 n+1$, then $M(n)$ admits infinitely many $(2 n+1)$-atomic representing measures (supported in the above mentioned circle).

When we consider $M(n)$ with a relation $\bar{Z} Z=a 1+b Z+c \bar{Z}+d Z^{2}(d \neq 0)$, the variety condition $\operatorname{card} \mathcal{V}(\gamma) \geq \operatorname{rank} M(n)$ appears for the first time.

Theorem 1.3. ([Fia3, Theorem 1.1, Theorem 1.3]) Suppose $M(n)$ is positive and recursively generated, and $\bar{Z} Z=a 1+b Z+c \bar{Z}+d Z^{2}, d \neq 0$. The following are equivalent for $\gamma \equiv \gamma^{(2 n)}$ :

(i) $\gamma$ admits a representing measure;

(ii) $\gamma$ admits a rank $M(n)$-atomic representing measure;

(iii) there exists $\gamma_{n, n+1} \in \mathbb{C}$ such that

$$
\gamma_{n+1, n} \equiv \bar{\gamma}_{n, n+1}=a \gamma_{n, n-1}+b \gamma_{n, n}+c \gamma_{n+1, n-1}+d \gamma_{n, n+1}
$$

(iv) $\operatorname{rank} M(n)(\gamma) \leq \operatorname{card} \mathcal{V}(\gamma)$. 
(For $n=2$, we established in [CuFi6, Theorem 3.1] the equivalence of (ii), (iii) and (i') $\gamma$ admits a finitely atomic representing measure.)

To complete the analysis of the truncated moment problem with a degree 2 relation, it would suffice to treat the case when $M(n)$ is positive and recursively generated, $\left\{1, Z, \bar{Z}, Z^{2}, \bar{Z} Z\right\}$ is independent, and $\bar{Z}^{2} \in\left\langle 1, Z, \bar{Z}, Z^{2}, \bar{Z} Z\right\rangle$. A direct attack on this problem seems difficult. Instead, we recall from [CuFi6, Proposition 1.7 and Section 5] that the truncated moment problem with a degree 2 relation can be reduced to the case of a real moment matrix with a column relation corresponding to one of the basic conics, $x^{2}+y^{2}=1, y=x^{2}, x y=1$, or $x y=0$. The circle case is subsumed by Theorem 1.2 and the hyperbolic cases will be analyzed in [CuFi8]. Our aim here is to treat the case $y=x^{2}$; our main result, which follows, parallels Theorem 1.3 in its use of the variety condition.

Theorem 1.4. Let $\beta \equiv \beta^{(2 n)}: \beta_{00}, \beta_{01}, \beta_{10}, \ldots, \beta_{0,2 n}, \ldots, \beta_{2 n, 0}$ be a family of real numbers, $\beta_{00}>0$, and let $\mathcal{M}(n)$ be the associated moment matrix. $\beta$ admits a representing measure supported in $y=x^{2}$ if and only if $\mathcal{M}(n)$ is positive, recursively generated, satisfies $Y=X^{2}$, and $\operatorname{rank} \mathcal{M}(n) \leq \operatorname{card} \mathcal{V}(\beta)$. In this case, $\mathcal{M}(n)$ admits a flat extension $\mathcal{M}(n+1)$ and $\beta$ admits a $\operatorname{rank} \mathcal{M}(n)$-atomic (minimal) representing measure.

Corollary 1.5. If $p(x, y)=0$ is a parabola, then $p$ satisfies $\left(A_{n}^{\prime}\right)$ for each $n \geq 2$.

Example 1.6. We define

$$
\mathcal{M}(3)(\beta):=\left(\begin{array}{cccccccccc}
1 & 0 & a & a & 0 & b & 0 & b & 0 & c \\
0 & a & 0 & 0 & b & 0 & b & 0 & c & 0 \\
a & 0 & b & b & 0 & c & 0 & c & 0 & d \\
a & 0 & b & b & 0 & c & 0 & c & 0 & d \\
0 & b & 0 & 0 & c & 0 & c & 0 & d & 0 \\
b & 0 & c & c & 0 & d & 0 & d & 0 & e \\
0 & b & 0 & 0 & c & 0 & c & 0 & d & 0 \\
b & 0 & c & c & 0 & d & 0 & d & 0 & e \\
0 & c & 0 & 0 & d & 0 & d & 0 & e & 0 \\
c & 0 & d & d & 0 & e & 0 & e & 0 & f
\end{array}\right) .
$$

With $a>0, b>a^{2}, c>\frac{b^{2}}{a}$, and $d>\frac{b^{3}-2 a b c+c^{2}}{b-a^{2}}$, we have $\mathcal{M}(2) \geq 0$ and $\operatorname{rank} \mathcal{M}(2)=5$. With

$$
e=\frac{-c^{3}+2 b c d-a d^{2}}{b^{2}-a c} \text { and } f \geq F:=-\frac{b c^{4}-b^{2} c^{2} d-2 a c^{3} d-b^{3} d^{2}+4 a b c d^{2}-a^{2} d^{2}}{\left(b^{2}-a c\right)^{2}},
$$

we have $\mathcal{M}(3) \geq 0, Y=X^{2}$ in $\mathcal{C}_{\mathcal{M}(3)},\left\{1, X, Y, Y X, Y^{2}\right\}$ linearly independent in $\mathcal{C}_{\mathcal{M}(3)}$, and $Y^{2} X=$ $\sigma X+\tau Y X$, where $\sigma:=\frac{-c^{2}+b d}{b^{2}-a c}$ and $\tau:=\frac{b c-a d}{b^{2}-a c}$. Thus, $5 \leq \operatorname{rank} \mathcal{M}(3) \leq 6$; in particular, $\mathcal{M}(3)$ is recursively generated. We will show by a direct calculation that $\beta^{(6)}$ has a representing measure if and only if card $\mathcal{V}(\beta) \geq \operatorname{rank} \mathcal{M}(3)$ or, equivalently, if and only if $f=F$.

To compute $\mathcal{V}(\beta)$, consider the relation $Y^{2} X=\sigma X+\tau Y X$. At the base space level for a potential representing measure, we must have $x\left(y^{2}-\tau y-\sigma\right)=x y^{2}-(\sigma x+\tau y x)=0$, and we assert that $\Psi(y):=y^{2}-\tau y-\sigma$ has distinct positive roots. For, consider the discriminant

$$
\tau^{2}+4 \sigma \equiv \lambda(d):=a^{2} d^{2}+\left(4 b^{3}-6 a b c\right) d+4 a c^{3}-3 b^{2} c^{2} .
$$

The equation $\lambda(d)=0$ has no real roots since its discriminant is $\left(4 b^{3}-6 a b c\right)^{2}-4 a^{2}\left(4 a c^{3}-3 b^{2} c^{2}\right)=$ $16\left(b^{2}-a c\right)^{3}<0$. Since

$$
\lambda(0) \equiv 4 a c^{3}-3 b^{2} c^{2}=c^{2}\left(4 a c-3 b^{2}\right)>3 c^{2}\left(a c-b^{2}\right)>0,
$$


we obtain that $\lambda(d)>0$ for all real $d$. This shows that $\Psi$ has distinct real roots. To show that these roots are positive, it suffices to verify that $-\sigma(=\Psi(0))>0$ and that $\tau>0$ (since the minimum for $\Psi$ occurs at $\left.\frac{\tau}{2}>0\right)$. To this end, note that since $b^{4}-2 a b^{2} c+a^{2} c^{2}=\left(b^{2}-a c\right)^{2}>0$, then $\frac{b^{3}-2 a b c+c^{2}}{b-a^{2}}>\frac{c^{2}}{b}$, whence $d>\frac{c^{2}}{b}$. Thus, $\sigma \equiv \frac{-c^{2}+b d}{b^{2}-a c}<0$. Also, $a c>b^{2}$, so $d>\frac{c^{2}}{b}>\frac{b c}{a}$, whence $\tau \equiv \frac{b c-a d}{b^{2}-a c}>0$.

Since $y^{2}-\tau y-\sigma=0$ has distinct positive roots, say $y_{1}$ and $y_{2}$, it follows that

$$
\begin{aligned}
\mathcal{V}(\beta) & \subseteq V:=\left\{(x, y): y=x^{2}\right\} \bigcap\left\{(x, y): x\left(y^{2}-\tau y-\sigma\right)=0\right\} \\
& =\left\{(0,0),\left(\sqrt{y_{1}}, y_{1}\right),\left(-\sqrt{y_{1}}, y_{1}\right),\left(\sqrt{y_{2}}, y_{2}\right),\left(-\sqrt{y_{2}}, y_{2}\right)\right\}
\end{aligned}
$$

If $f>F$, then $\operatorname{rank} \mathcal{M}(3)=6>5 \geq \operatorname{card} \mathcal{V}(\beta)$, so $\beta$ admits no representing measure. Suppose now that $f=F$. Then $Y^{3}=\sigma Y+\tau Y^{2}$ and $\operatorname{rank} \mathcal{M}(3)=5$. One might expect that this new column relation would cause $\mathcal{V}(\beta)$ to be a proper subset of $V$, but this is not the case. Indeed, since $Y^{3}=\sigma Y+\tau Y^{2}$ corresponds to $y\left(y^{2}-\tau y-\sigma\right)=0$, then $\mathcal{V}(\beta)=V \bigcap\left\{(x, y): y\left(y^{2}-\tau y-\sigma\right)=0\right\}=V$, whence $\operatorname{card} \mathcal{V}(\beta)=\operatorname{rank} \mathcal{M}(3)=5$. In this case, since $\mathcal{M}(3)$ is flat, then $\beta$ admits a unique representing measure, which is 5-atomic.

For a numerical example, let $a=1, b=2, c=5, d=14, e=41$ and $f=122$. Then $\mathcal{V}(\beta)=$ $\{(0,0),(-1,1),(1,1),(-\sqrt{3}, 3),(\sqrt{3}, 3)\}$, and the corresponding 5-atomic representing measure is $\mu=$ $\frac{1}{3} \delta_{(0,0)}+\frac{1}{4}\left(\delta_{(-1,1)}+\delta_{(1,1)}\right)+\frac{1}{12}\left(\delta_{(-\sqrt{3}, 3)}+\delta_{(\sqrt{3}, 3)}\right)$.

The remainder of this section is devoted to notation and basic results concerning real moment matrices. Given a collection $\beta^{(2 n)}: \beta_{00}, \beta_{01}, \beta_{10}, \ldots, \beta_{0,2 n}, \ldots, \beta_{2 n, 0}$, we can build an associated moment matrix $\mathcal{M}(n)(\beta):=(\mathcal{M}[i, j](\beta))_{i, j=0}^{n}$, where

$$
\mathcal{M}[i, j](\beta):=\left(\begin{array}{cccc}
\beta_{0, i+j} & \beta_{1, i+j-1} & \cdots & \beta_{j, i} \\
\beta_{1, i+j-1} & \beta_{2, i+j-2} & \cdots & \beta_{j+1, i-1} \\
\vdots & \vdots & \ddots & \vdots \\
\beta_{i, j} & \beta_{i+1, j-1} & \cdots & \beta_{i+j, 0}
\end{array}\right) .
$$

We denote the successive rows and columns of $\mathcal{M}(n)(\beta)$ by $1, X, Y, X^{2}, Y X, Y^{2}, \ldots, X^{n}, \ldots, Y^{n}$; observe that each block $\mathcal{M}[i, j](\beta)$ is of Hankel type, i.e., constant on cross-diagonals. The matrix $\mathcal{M} \equiv \mathcal{M}(n)(\beta)$ gives rise to a semi-inner product that we next describe. Let $\mathcal{P}_{n}^{\mathbb{R}}$ denote the real polynomials $q(x, y) \equiv \sum a_{i j} y^{i} x^{j}$ of total degree at most $n$, and for $q \in \mathcal{P}_{n}^{\mathbb{R}}$, let $\tilde{q}:=\left(a_{i j}\right)$ denote the coefficient vector of $q$ with respect to the basis $\left\{y^{i} x^{j}\right\}_{0 \leq i+j \leq n}$ of $\mathcal{P}_{n}^{\mathbb{R}}$ (ordered lexicographically: $1, x$, $\left.y, x^{2}, y x, y^{2}, \ldots, x^{n}, \ldots, y^{n}\right)$. For $r, s \in \mathcal{P}_{n}^{\mathbb{R}}$, define $\langle r, s\rangle_{\mathcal{M}(n)}:=(\mathcal{M}(n) \widetilde{r}, \widetilde{s}) ;\langle\cdot, \cdot\rangle_{\mathcal{M}(n)}$ is a semiinner product on $\mathcal{P}_{n}^{\mathbb{R}}$. For $p \in \mathcal{P}_{2 n}^{\mathbb{R}}, p(x, y) \equiv \sum_{0 \leq i+j \leq 2 n} b_{i j} y^{i} x^{j}$, let $L_{\beta}(p):=\sum_{0 \leq i+j \leq 2 n} b_{i j} \beta_{i j}$; $L_{\beta}$ is the Riesz functional associated to $\beta$. Note that $\mathcal{M}(n)(\beta)$ is the unique matrix (of size $\left.m(n):=\frac{(n+1)(n+2)}{2}\right)$ such that

$$
\langle\mathcal{M}(n) \hat{f}, \hat{g}\rangle=L_{\beta}(f g) \quad\left(f, g \in \mathcal{P}_{n}^{\mathbb{R}}\right)
$$

Note also that the row $Y^{k} X^{\ell}$, column $Y^{i} X^{j}$ entry of $\mathcal{M}(n)$ is equal to

$$
\left\langle\mathcal{M}(n) \widetilde{y^{i} x^{j}}, \widetilde{y^{k} x^{\ell}}\right\rangle=L_{\beta}\left(y^{i+k} x^{j+\ell}\right)=\beta_{i+k, j+\ell}
$$


For example, with $n=1$, the Quadratic Moment Problem for $\beta^{(2)}: \beta_{00}, \beta_{01}, \beta_{10}, \beta_{02}, \beta_{11}, \beta_{20}$ corresponds to

$$
\mathcal{M}(1)=\left(\begin{array}{lll}
\beta_{00} & \beta_{01} & \beta_{10} \\
\beta_{01} & \beta_{02} & \beta_{11} \\
\beta_{10} & \beta_{11} & \beta_{20}
\end{array}\right),
$$

and for $n=2$, the Quartic Moment Problem for $\beta^{(4)}$ corresponds to

$$
\mathcal{M}(2)=\left(\begin{array}{llllll}
\beta_{00} & \beta_{01} & \beta_{10} & \beta_{02} & \beta_{11} & \beta_{20} \\
\beta_{01} & \beta_{02} & \beta_{11} & \beta_{03} & \beta_{12} & \beta_{21} \\
\beta_{10} & \beta_{11} & \beta_{20} & \beta_{12} & \beta_{21} & \beta_{30} \\
\beta_{02} & \beta_{03} & \beta_{12} & \beta_{04} & \beta_{13} & \beta_{22} \\
\beta_{11} & \beta_{12} & \beta_{21} & \beta_{13} & \beta_{22} & \beta_{31} \\
\beta_{20} & \beta_{21} & \beta_{30} & \beta_{22} & \beta_{31} & \beta_{40}
\end{array}\right) .
$$

If $\beta$ admits a representing measure $\mu$, then for $f \in \mathcal{P}_{n}^{\mathbb{R}}$,

$$
\langle\mathcal{M}(n) \tilde{f}, \tilde{f}\rangle=L_{\beta}\left(f^{2}\right)=\int f^{2} d \mu \geq 0,
$$

whence $\mathcal{M}(n) \geq 0$.

Recall that $\mathcal{M}(n)$ has size $m(n) \equiv \frac{(n+1)(n+2)}{2}$. For any matrix $M$ of this size, $[M]_{k}$ denotes the compression of $M$ to the first $k$ rows and columns and $\left\langle Y^{i} X^{j}, Y^{k} X^{l}\right\rangle_{M}$ denotes the entry in row $Y^{k} X^{l}$ and column $Y^{i} X^{j}$. Similarly, for a vector $\mathbf{v},[\mathbf{v}]_{k}$ denotes the compression of $\mathbf{v}$ to the first $k$ entries. We also consider compressions of $M$ to a set $E$ of rows and columns, and denote such compressions by $[M]_{E}$. In the sequel, unless otherwise stated, we always assume that $\beta^{(2 n)}$ satisfies $\beta_{00}=1$; this amounts to rescaling the total mass, and has no effect as to existence, uniqueness or support of representing measures.

We next recall from [CuFi1] some additional necessary conditions for the existence of representing measures. Let $\mathcal{C}_{\mathcal{M}(n)}$ denote the column space of $\mathcal{M}(n)$, i.e., $\mathcal{C}_{\mathcal{M}(n)}=\left\langle 1, X, Y, \ldots, X^{n}, \ldots, Y^{n}\right\rangle \subseteq$ $\mathbb{R}^{m(n)}$. For $p \in \mathcal{P}_{n}^{\mathbb{R}}, p \equiv \sum a_{i j} y^{i} x^{j}$, we define $p(X, Y) \in \mathcal{C}_{\mathcal{M}(n)}$ by $p(X, Y):=\sum a_{i j} Y^{i} X^{j}$. Given $p(X, Y) \in \mathcal{C}_{\mathcal{M}(n)}$ with $\operatorname{deg} p \leq n-1$, we let $p(X, Y) X:=(x p)(X, Y)$ and $p(X, Y) Y:=(y p)(X, Y)$. If $\mu$ is a representing measure for $\beta$, then for $p \in \mathcal{P}_{n}^{\mathbb{R}}$,

$$
p(X, Y)=0 \Leftrightarrow \operatorname{supp} \mu \subseteq \mathcal{Z}(p):=\left\{(x, y) \in \mathbb{R}^{2}: p(x, y)=0\right\}
$$

([CuFi1, Proposition 3.1]). As a consequence, the following condition holds: if $\mu$ is a representing measure for $\beta$, then

$$
\text { card } \operatorname{supp} \mu \geq \operatorname{rank} \mathcal{M}(n) \quad([\text { CuFi1, Corollary 3.5]) }
$$

In [CuFi1] we actually formulated the last two results only for the case of TCMP, where $M(n)(\gamma)$ admits column relations of the form $p(Z, \bar{Z})=0$; the validity of these equivalent statements for the real moment problem follows from [CuFi4, Proposition 1.12]. Similarly, the main result of [CuFi1] ([CuFi1, Theorem 5.13]), properly translated to the context of real moment matrices, shows that $\beta^{(2 n)}$ admits a rank $\mathcal{M}(n)$-atomic (minimal) representing measure if and only if $\mathcal{M}(n) \geq 0$ and $\mathcal{M}(n)$ admits an extension to a (necessarily positive) moment matrix $\mathcal{M}(n+1)$ satisfying $\operatorname{rank} \mathcal{M}(n+1)=\operatorname{rank} \mathcal{M}(n)$.

A theorem of Smul'jan [Smu] shows that a block matrix

$$
M=\left(\begin{array}{cc}
A & B \\
B^{*} & C
\end{array}\right)
$$


is positive if and only if (i) $A \geq 0$, (ii) there exists a matrix $W$ such that $B=A W$, and (iii) $C \geq W^{*} A W$ (since $A=A^{*}, W^{*} A W$ is independent of $W$ satisfying $B=A W$ ). Note also that if $M \geq 0$, then $\operatorname{rank} M=\operatorname{rank} A$ if and only if $C=W^{*} A W$; conversely, if $A \geq 0$ and there exists $W$ such that $B=A W$ and $C=W^{*} A W$, then $M \geq 0$ and $\operatorname{rank} M=\operatorname{rank} A$. In the sequel, for $A \geq 0$, we refer to $M$ as an extension of $A$, and as a flat extension if $\operatorname{rank} M=\operatorname{rank} A$. Thus, a flat extension of a positive matrix $A$ is completely determined by a choice of block $B$ satisfying $B=A W$ and $C=W^{*} A W$ for some matrix $W$; we denote such an extension by $[A ; B]$. An immediate consequence of the condition $\operatorname{rank} M=\operatorname{rank} A$ and of the Extension Principle [Fia1, Proposition 2.4] is that any column dependence relations valid in $\left(\begin{array}{ll}A & B\end{array}\right)$ extend to the columns of $M$.

For an $(n+1) \times(n+2)$ moment matrix block $B_{n, n+1}$, representing "new moments" of degree $2 n+1$ for a prospective representing measure of $\beta^{(2 n)}$, let

$$
B(n+1):=\left(\begin{array}{c}
B_{0, n+1} \\
\vdots \\
B_{n-1, n+1} \\
B_{n, n+1}
\end{array}\right) .
$$

By Smul'jan's theorem, $\mathcal{M}(n) \geq 0$ admits a (necessarily positive) flat extension

$$
[\mathcal{M}(n): B]=\left(\begin{array}{cc}
\mathcal{M}(n) & B \\
B^{*} & C
\end{array}\right)
$$

in the form of a moment matrix $\mathcal{M}(n+1)$ if and only if

$$
\begin{aligned}
& B=B(n+1) \text { and } B=\mathcal{M}(n) W \text { for some } W \\
& \text { (i.e., } \operatorname{Ran} B \subseteq \operatorname{Ran} \mathcal{M}(n)[\mathrm{Dou}]) \text {; and } \\
& C:=W^{*} \mathcal{M}(n) W \text { is Hankel } \\
& \text { (i.e., } \left.C \text { has the form of a moment matrix block } B_{n+1, n+1}\right) \text {. }
\end{aligned}
$$

We also recall from [CuFi1] and [Fia1] that $\mathcal{M}(n) \geq 0$ is recursively generated if the following property holds:

$$
p, q, p q \in \mathcal{P}_{n}^{\mathbb{R}}, p(X, Y)=0 \quad \Longrightarrow \quad(p q)(X, Y)=0 .
$$

If $\mathcal{M}(n) \geq 0$ admits a flat extension $\mathcal{M}(n+1)$, then $\mathcal{M}(n+1)$, and all of its successive flat extensions $\mathcal{M}(n+1+d)$ are recursively generated [CuFi1, Remark 3.15-ii)]. More generally, if $\beta^{(2 n)}$ admits a representing measure, then $\mathcal{M}(n)$ is recursively generated [CuFi1, Corollary 3.4].

Acknowledgment. Some examples, and portions of the proofs of some results in this paper, were obtained using calculations with the software tool Mathematica [Wol].

\section{The Parabolic Moment Problem}

Recall Theorem 1.4: $\beta^{(2 n)}$ has a representing measure supported in the parabola $y=x^{2}$ if and only if $\mathcal{M}(n)(\beta)$ is positive, recursively generated, has the column relation $Y=X^{2}$, and $\operatorname{rank} \mathcal{M}(n)(\beta) \leq$ $\operatorname{card} \mathcal{V}(\beta)$. The necessity of the conditions is clear from Section 1. In this section, we prove the main step toward sufficiency, which is the following result.

Theorem 2.1. Let $\beta \equiv \beta^{(2 n)}: \beta_{00}, \beta_{01}, \beta_{10}, \ldots, \beta_{0,2 n}, \ldots, \beta_{2 n, 0}$ be a family of real numbers, $\beta_{00}>0$, and let $\mathcal{M}(n)$ be the associated moment matrix. Assume that $\mathcal{M}(n)$ is positive, recursively generated, and satisfies $Y=X^{2}$ and rank $\mathcal{M}(n) \leq \operatorname{card} \mathcal{V}(\beta)$. Then $\mathcal{M}(n)$ admits a flat extension $\mathcal{M}(n+1)$.

In Section 3 we will use Theorem 2.1 to prove the following result, which includes the sufficiency part of Theorem 1.4. 
Theorem 2.2. Let $\beta \equiv \beta^{(2 n)}: \beta_{00}, \beta_{01}, \beta_{10}, \ldots, \beta_{0,2 n}, \ldots, \beta_{2 n, 0}$ be a family of real numbers, $\beta_{00}>0$, and let $\mathcal{M}(n)$ be the associated moment matrix. Assume that $\mathcal{M}(n)$ is positive, recursively generated, and satisfies $Y=X^{2}$. The following statements are equivalent.

(i) $\beta$ admits a representing measure (necessarily supported in $y=x^{2}$ ).

(ii) $\beta$ admits a representing measure with moments of order up to $2 n+2$ (necessarily supported in $\left.y=x^{2}\right)$.

(iii) $\beta$ admits a rank $\mathcal{M}(n)$-atomic representing measure (necessarily supported in $y=x^{2}$ ).

(iv) $\mathcal{M}(n)$ admits a positive, recursively generated extension $\mathcal{M}(n+1)$.

(v) $\mathcal{M}(n)$ admits a flat extension $\mathcal{M}(n+1)$.

(vi) $\operatorname{rank} \mathcal{M}(n) \leq \operatorname{card} \mathcal{V}(\beta)$.

In Section 5 we will use Theorem 2.2 to obtain a new proof (for parabolas) of J. Stochel's solution to the full moment problem on curves of degree $\leq 2$ (cf [Sto1]); we may formulate this result as follows.

Theorem 2.3. (cf. [Sto1]) The sequence $\beta \equiv \beta^{(\infty)}$ admits a representing measure supported in the parabola $y=x^{2}$ if and only if $\mathcal{M}(\infty) \geq 0$ and $Y=X^{2}$.

Of course, Theorems $2.1-2.3$ extend to general parabolas in the plane.

By [CuFi2, Theorem 2.1], we know that $\mathcal{M}(n)$ admits flat extensions when $\{1, X, Y\}$ is linearly dependent in $\mathcal{C}_{\mathcal{M}(n)}$. Thus, hereafter we will assume that $\{1, X, Y\}$ is linearly independent. Toward the proof of Theorem 2.1, we begin with an elementary lemma that exploits the fact that $\mathcal{M}(n)$ is recursively generated. For $1 \leq k \leq n$ let

$$
\mathcal{S}_{n}(k):=\left\{1, X, Y, Y X, Y^{2}, Y^{2} X, Y^{3}, \ldots, Y^{k-1} X, Y^{k}\right\} \subseteq \mathcal{C}_{\mathcal{M}(n)} .
$$

Lemma 2.4. For $n \geq 2$, let $\mathcal{M}(n)$ be positive and recursively generated, and assume that $Y=X^{2}$. Then $\mathcal{S}_{n}(n)$ spans $\mathcal{C}_{\mathcal{M}(n)}$, and therefore rank $\mathcal{M}(n) \leq 2 n+1 ;$ moreover, each column of $\mathcal{M}(n)$ is equal to a column in $\mathcal{S}_{n}(n)$.

Proof. The proof is by induction on $n \geq 2$. For $n=2$ the statement is clearly true, so assume it holds for $n=k$. Suppose $\mathcal{M}(k+1)$ is positive and recursively generated, with $Y=X^{2}$ in $\mathcal{C}_{\mathcal{M}(k+1)}$. Consider a column in $\mathcal{M}(k+1)$ of the form $Y^{k+1-j} X^{j}$, with $2 \leq j \leq k+1$. Let $q(x, y):=y-x^{2}$ and let $p_{i j}(x, y):=y^{i} x^{j}$, so that $Y^{k+1-j} X^{j}=p_{k+1-j, j}(X, Y)$. Also, let $r_{i j}(x, y):=y^{k+2-j} x^{j-2}-y^{k+1-j} x^{j}$. Since $j \geq 2$, it is straightforward that $r_{i j}(x, y) \equiv y^{k+1-j} x^{j-2}\left(y-x^{2}\right)=p_{k+1-j, j-2}(x, y) q(x, y)$. Since $\mathcal{M}(k+1)$ is recursively generated and $q(X, Y)=0$, it follows that $r_{i j}(X, Y)=0$, that is, $Y^{k+1-j} X^{j}=Y^{k+2-j} X^{j-2}$ in $\mathcal{C}_{\mathcal{M}(k+1)}$. By induction, $\left[Y^{k+2-j} X^{j-2}\right]_{m(k)} \in$ lin.span $\mathcal{S}_{k}(k)$, and since $\mathcal{M}(k+1) \geq 0$, it follows from the Extension Principle [Fia1, Proposition 2.4] that $Y^{k+2-j} X^{j-2} \in$ lin.span $\mathcal{S}_{k+1}(k)$. Thus $Y^{k+1-j} X^{j}\left(=Y^{k+2-j} X^{j-2}\right) \in$ lin.span $\mathcal{S}_{k+1}(k) \subseteq$ lin.span $\mathcal{S}_{k+1}(k+1)$, as desired.

We next divide the proof of Theorem 2.1 into five cases, based on possible dependence relations among the elements of $\mathcal{S}_{n}(n)$. Section 4 contains examples illustrating these cases. In the sequel, unless stated otherwise, we are always assuming that $\mathcal{M}(n)$ is positive, recursively generated, $\{1, X, Y\}$ is linearly independent, $Y=X^{2}$, and $\operatorname{rank} \mathcal{M}(n) \leq \operatorname{card} \mathcal{V}(\beta)$.

Proposition 2.5. (Case I: For some $k, 2 \leq k<n-1, \mathcal{S}_{n}(k)$ is linearly independent and $Y^{k} X \in$ lin.span $\left.\mathcal{S}_{n}(k)\right)$ Assume that $\mathcal{M}(n)(\beta)$ is positive, recursively generated, and satisfies $Y=X^{2}$. In $\mathcal{S}_{n}(n)$, assume that the first dependence relation occurs at $Y^{k} X$, with $2 \leq k<n-1$. Then $\mathcal{M}(n)$ is flat and, a fortiori, it admits a unique flat extension $\mathcal{M}(n+1)$. 
Proof. Write

$$
Y^{k} X=p_{k}(Y)+q_{k-1}(Y) X
$$

where $\operatorname{deg} p_{k} \leq k, \operatorname{deg} q_{k-1} \leq k-1$. Since $Y^{k} X$ corresponds to a monomial of degree at most $n-1$, and since $Y=X^{2}$ and $\mathcal{M}(n)$ is recursively generated, we must have $Y^{k+1}=p_{k}(Y) X+q_{k-1}(Y) Y$. Substituting from (2.1) for the $Y^{k} X$ term in $p_{k}(Y) X$, we see that both $Y^{k} X$ and $Y^{k+1}$ are linear combinations of columns corresponding to monomials of degree at most $k$. It now follows from recursiveness and from Lemma 2.4 that $\mathcal{M}(n)$ is flat, so there exists a (unique) flat extension $\mathcal{M}(n+1)[\mathrm{CuFi1}$, Theorem 5.4].

Proposition 2.6. (Case II: The first dependence relation occurs at a column of the form $Y^{k}$ with $k<n)$ Assume that $\mathcal{M}(n)(\beta)$ is positive, recursively generated, and satisfies $Y=X^{2}$. In $\mathcal{S}_{n}(n)$, assume that $Y^{k}$ is the location of the first dependence relation, with $k<n$. Then $\mathcal{M}(n)$ is flat, and thus admits a unique flat extension $\mathcal{M}(n+1)$.

Proof. Write

$$
Y^{k}=p_{k-1}(Y)+q_{k-1}(Y) X
$$

where $\operatorname{deg} p_{k-1}, \operatorname{deg} q_{k-1} \leq k-1$. Since $k<n, Y=X^{2}$, and $\mathcal{M}(n)$ is recursively generated, we must have $Y^{k} X=p_{k-1}(Y) X+q_{k-1}(Y) Y$. We thus see that $Y^{k} X$ is a linear combination of columns in $\mathcal{S}_{n}(k)$. On the other hand, from (2.2) it follows that $Y^{k+1}=p_{k-1}(Y) Y+q_{k-1}(Y) Y X$. If $\operatorname{deg} q_{k-1}=k-1$, we may apply $(2.2)$ to the $Y^{k}$ term in $q_{k-1}(Y) Y$, and also use $Y=X^{2}$, to see that $Y^{k+1}$ is a linear combination of columns in $\mathcal{S}_{n}(k)$. It now follows (via recursiveness and Lemma 2.4) that $\mathcal{M}(n)$ is flat; thus $\mathcal{M}(n)$ admits a unique flat extension $\mathcal{M}(n+1)$.

Proposition 2.7. (Case III: The first dependence relation occurs at a column of the form $Y^{n-1} X$ ) Assume that $\mathcal{M}(n)(\beta)$ is positive, recursively generated, and satisfies $Y=X^{2}$ and $\operatorname{rank} \mathcal{M}(n) \leq$ card $\mathcal{V}(\beta)$. In $\mathcal{S}_{n}(n)$, assume that the first dependence relation occurs at $Y^{n-1} X$. Then $\mathcal{M}(n)$ is flat, and thus admits a unique flat extension $\mathcal{M}(n+1)$.

Proof. Write $Y^{n-1} X=p_{n-1}(Y)+q_{n-2}(Y) X$, with $\operatorname{deg} p_{n-1} \leq n-1$, $\operatorname{deg} q_{n-2} \leq n-2$, and let $r(x, y):=y^{n-1} x-\left(p_{n-1}(y)+q_{n-2}(y) x\right)$ and $s(x, y):=y-x^{2}$. It follows that $\mathcal{V}(\beta) \subseteq \mathcal{Z}(r) \bigcap \mathcal{Z}(s)$. Now observe that if we substitute $y=x^{2}$ in $r(x, y)=0$, we obtain a polynomial equation in $x$ of degree at most $2 n-1$. It then follows that card $\mathcal{V}(\beta) \leq 2 n-1$, so that rank $\mathcal{M}(n) \leq$ card $\mathcal{V}(\beta) \leq 2 n-1$. Then $\mathcal{S}_{n}(n-1) \equiv\left\{1, X, Y, Y X, Y^{2}, Y^{2} X, Y^{3}, \ldots, Y^{n-2} X, Y^{n-1}\right\}$ is a basis for $\overline{\mathcal{C}}_{\mathcal{M}(n)}$, whence $Y^{n}$ is a linear combination of the columns in $\mathcal{S}_{n}(n-1)$. Since, by recursiveness, the columns $Y^{i} X^{j}$, with $i+j=n$ and $j \geq 2$, coincide with columns of lower degree, it now follows that $\mathcal{M}(n)$ is flat, and thus admits a unique flat extension $\mathcal{M}(n+1)$.

Proposition 2.8. (Case IV: The first dependence relation occurs at $Y^{n}$ ) Assume that $\mathcal{M}(n)(\beta)$ is positive, recursively generated, and satisfies $Y=X^{2}$. In $\mathcal{S}_{n}(n)$, assume that the first dependence relation occurs at $Y^{n}$. Then $\mathcal{M}(n)$ admits a unique flat extension $\mathcal{M}(n+1)$.

Under the hypotheses of Proposition 2.8, write

$$
Y^{n}=p_{n-1}(Y)+q_{n-1}(Y) X
$$

where $\operatorname{deg} p_{n-1}, \operatorname{deg} q_{n-1} \leq n-1$. (The expression $p_{n-1}(Y)+q_{n-1}(Y) X$ is shorthand notation for $\left(p_{n-1}+x q_{n-1}\right)(X, Y)$.) To build a flat extension $\mathcal{M}(n+1)$, we define the first $n$ columns of a prospective $B$ block by exploiting the relation $Y=X^{2}$, as follows: $X^{n+1}:=Y X^{n-1}, Y X^{n}:=$ $Y^{2} X^{n-2}, \ldots, Y^{n-1} X^{2}:=Y^{n}$. Also, using (2.3), we let

$$
Y^{n} X:=p_{n-1}(Y) X+q_{n-1}(Y) Y \in \mathcal{C}_{\mathcal{M}(n)}
$$


(where $\left.p_{n-1}(Y) X+q_{n-1}(Y) Y=\left(x p_{n-1}+y q_{n-1}\right)(X, Y)\right)$ and, using $(2.3)$ and (2.4), we let

$$
Y^{n+1}:=p_{n-1}(Y) Y+q_{n-1}(Y) Y X \in \mathcal{C}_{\mathcal{M}(n)}
$$

(where $\left.p_{n-1}(Y) Y+q_{n-1}(Y) Y X=\left(y p_{n-1}+x y q_{n-1}\right)(X, Y)\right)$. (Observe that these defining relations are all required if one is to obtain a positive recursively generated moment matrix extension for $\mathcal{M}(n)$.) Since the columns $(2.3)$ - $(2.5)$ belong to $\mathcal{C}_{\mathcal{M}(n)}$, we have $B=\mathcal{M}(n) W$ for some matrix $W$. Thus, a flat extension $\mathcal{M}:=[\mathcal{M}(n) ; B]$ is uniquely determined by defining the $C$-block as $C:=W^{*} \mathcal{M}(n) W$ (cf. Section 1). To complete the proof that $\mathcal{M}$ is a moment matrix $\mathcal{M}(n+1)$, it suffices to show that block $B$ is of the form $\left(B_{i, n+1}\right)_{i=0}^{n}$ and that block $C$ is of the form $B_{n+1, n+1}$.

To this end, we require some additional notation and several preliminary results. Recall that for $i+j, k+\ell \leq n$, we have

$$
\left\langle Y^{i} X^{j}, Y^{k} X^{\ell}\right\rangle \equiv\left\langle Y^{i} X^{j}, Y^{k} X^{\ell}\right\rangle_{\mathcal{M}(n)}=\beta_{i+k, j+\ell} .
$$

For $p, q \in \mathcal{P}_{n}^{\mathbb{R}}, p(x, y) \equiv \sum_{0 \leq i+j \leq n} a_{i j} x^{j} y^{i}, q(x, y) \equiv \sum_{0 \leq i+j \leq n} b_{k, \ell} x^{\ell} y^{k}$, we define

$$
\langle p(X, Y), q(X, Y)\rangle:=\sum_{\substack{0 \leq i+j \leq n \\ 0 \leq k+\ell \leq n}} a_{i j} b_{k \ell}\left\langle Y^{i} X^{j}, Y^{k} X^{\ell}\right\rangle=\sum_{\substack{0 \leq i+j \leq n \\ 0 \leq k+\ell \leq n}} a_{i j} b_{k \ell} \beta_{i+k, j+\ell}
$$

The following result follows directly from the preceding definitions.

Lemma 2.9. (i) For $p, q \in \mathcal{P}_{n}^{\mathbb{R}}$,

$$
\langle p(X, Y), q(X, Y)\rangle=\langle q(X, Y), p(X, Y)\rangle .
$$

(ii) For $p, q \in \mathcal{P}_{n}^{\mathbb{R}}, i, j \geq 0, i+j \leq n$, and $\operatorname{deg} p, \operatorname{deg} q \leq n-(i+j)$,

$$
\left\langle p(X, Y) Y^{j} X^{i}, q(X, Y)\right\rangle=\left\langle p(X, Y), q(X, Y) Y^{j} X^{i}\right\rangle .
$$

(iii) If $p, q, r \in \mathcal{P}_{n}^{\mathbb{R}}$ with $p(X, Y)=q(X, Y)$ in $\mathcal{C}_{\mathcal{M}(n)}$, then $\langle r(X, Y), p(X, Y)\rangle=\langle r(X, Y), q(X, Y)\rangle$.

We next extend the notation $\langle p(X, Y), q(X, Y)\rangle$ to the case when $\operatorname{deg} p=n+1, \operatorname{deg} q \leq n$. Indeed, using the definitions of the columns of $B$, for $i, j \geq 0, i+j=n+1$, there exists $p_{i j} \in \mathcal{P}_{n}^{\mathbb{R}}$ with $Y^{i} X^{j}=p_{i j}(X, Y)$, and we define

$$
\left\langle Y^{i} X^{j}, q(X, Y)\right\rangle:=\left\langle p_{i j}(X, Y), q(X, Y)\right\rangle .
$$

Now if $p(x, y) \equiv \sum_{0 \leq k+\ell \leq n+1} a_{k \ell} x^{\ell} y^{k}$, we define

$$
\langle p(X, Y), q(X, Y)\rangle:=\sum_{0 \leq k+\ell \leq n+1} a_{k \ell}\left\langle Y^{k} X^{\ell}, q(X, Y)\right\rangle .
$$

It is easy to check that Lemma 2.9(iii) holds with $\operatorname{deg} r=n+1$.

Lemma 2.10. Assume $i+j=n+1, s \geq 2$, and $r+s \leq n$. Then

$$
\left\langle Y^{i} X^{j}, Y^{r} X^{s}\right\rangle=\left\langle Y^{i} X^{j}, Y^{r+1} X^{s-2}\right\rangle \text {. }
$$


Proof. Fix $i$ and $j$ with $i+j=n+1$. We know that there exists a polynomial $p \in \mathcal{P}_{n}^{\mathbb{R}}$ such that $Y^{i} X^{j}=p(X, Y) \equiv \sum_{k+\ell \leq n} a_{k, \ell} Y^{k} X^{\ell}$. Then

$$
\begin{aligned}
\left\langle Y^{i} X^{j}, Y^{r} X^{s}\right\rangle & =\sum_{k+\ell \leq n} a_{k, \ell}\left\langle Y^{k} X^{\ell}, Y^{r} X^{s}\right\rangle=\sum_{k+\ell \leq n} a_{k, \ell}\left\langle Y^{r} X^{s}, Y^{k} X\right. \\
& \text { (because } \mathcal{M}(n) \text { is self-adjoint) } \\
& =\sum_{k+\ell \leq n} a_{k, \ell}\left\langle Y^{r+1} X^{s-2}, Y^{k} X^{\ell}\right\rangle \quad \text { (using } Y=X^{2} \text { ) } \\
& =\sum_{k+\ell \leq n} a_{k, \ell}\left\langle Y^{k} X^{\ell}, Y^{r+1} X^{s-2}\right\rangle \\
& \text { (using again the self-adjointness of } \mathcal{M}(n)) \\
& =\left\langle Y^{i} X^{j}, Y^{r+1} X^{s-2}\right\rangle,
\end{aligned}
$$

as desired.

Corollary 2.11. Assume $i+j=n+1$, with $j \geq 1$, and assume that the Hankel property

$$
\left\langle Y^{i} X^{j}, Y^{r} X^{s}\right\rangle=\left\langle Y^{i+1} X^{j-1}, Y^{r-1} X^{s+1}\right\rangle
$$

holds for all $Y^{r} X^{s} \in \mathcal{S}_{n}(n)$ with $r \geq 1$. Then (2.7) holds for all $r$ and $s$ such that $1 \leq r+s \leq n$, $r \geq 1$.

Proof. Fix $i$ and $j$ with $i+j=n+1$. We do induction on $t:=r+s$, where $1 \leq r+s \leq n, r \geq 1$. For $t=1$ the result is clear, since $Y \in \mathcal{S}_{n}(n)$, and for $t=2$ the result follows from the fact that $Y X$ and $Y^{2}$ are in $\mathcal{S}_{n}(n)$. Assume the statement is true for $t=u \geq 2$, and consider the case $t=u+1$. For $Y^{r} X^{s}$ with $r+s=u+1$, we may assume $Y^{r} X^{s} \notin \mathcal{S}_{n}(n)$, whence $s \geq 2$. Now,

$$
\begin{aligned}
\left\langle Y^{i} X^{j}, Y^{r} X^{s}\right\rangle & =\left\langle Y^{i} X^{j}, Y^{r+1} X^{s-2}\right\rangle \quad(\text { by }(2.6)) \\
& =\left\langle Y^{i+1} X^{j-1}, Y^{r} X^{s-1}\right\rangle \quad \text { (by the inductive step and (2.7)) } \\
& =\left\langle Y^{i+1} X^{j-1}, Y^{r-1} X^{s+1}\right\rangle \quad \text { (by (2.6) again), }
\end{aligned}
$$

as desired.

Lemma 2.12. For $k=0, \ldots, n-2$,

$$
\left\langle Y^{n} X, Y^{k}\right\rangle=\left\langle Y^{n}, Y^{k} X\right\rangle .
$$

Proof.

$$
\begin{aligned}
\left\langle Y^{n} X, Y^{k}\right\rangle & \left.=\left\langle p_{n-1}(Y) X+q_{n-1}(Y) Y, Y^{k}\right\rangle \quad \text { (by }(2.4)\right) \\
& =\left\langle p_{n-1}(Y), Y^{k} X\right\rangle+\left\langle q_{n-1}(Y), Y^{k+1}\right\rangle \quad \text { (by Lemma 2.9(ii)) } \\
& =\left\langle p_{n-1}(Y), Y^{k} X\right\rangle+\left\langle q_{n-1}(Y), Y^{k} X^{2}\right\rangle \\
\text { (using } Y & \left.=X^{2}, \text { since } k \leq n-2, \text { and Lemma } 2.9(\mathrm{iii})\right) \\
& =\left\langle p_{n-1}(Y), Y^{k} X\right\rangle+\left\langle q_{n-1}(Y) X, Y^{k} X\right\rangle \quad \text { (by Lemma 2.9(ii)) } \\
& =\left\langle p_{n-1}(Y)+q_{n-1}(Y) X, Y^{k} X\right\rangle \\
& =\left\langle Y^{n}, Y^{k} X\right\rangle \quad(\text { by }(2.3)),
\end{aligned}
$$


as desired.

Proof of Proposition 2.8. The first part of the proof is devoted to showing that the $B$ block, as defined above, is of the form $\left\{B_{i, n+1}\right\}_{i=0}^{n}$. To this end, and since the first $n$ columns of $B$ are taken, as a package, from columns in $\mathcal{M}(n)$, it suffices to prove that the last three columns of $B$, namely $Y^{n-1} X^{2}, Y^{n} X$ and $Y^{n+1}$, satisfy the proper Hankel conditions. From Corollary 2.11, we can restrict attention to rows corresponding to monomials of the form $Y^{k} X(k=1, \ldots, n-1)$ and $Y^{k+1}$, for $k=0, \ldots, n-1$. We shall establish that

$$
\left\{\begin{array}{cc}
\text { (i) }\left\langle Y^{n-1} X^{2}, Y^{k} X\right\rangle=\left\langle Y^{n} X, Y^{k-1} X^{2}\right\rangle & (1 \leq k \leq n-1) \\
\text { (ii) }\left\langle Y^{n-1} X^{2}, Y^{k+1}\right\rangle=\left\langle Y^{n} X, Y^{k} X\right\rangle & (0 \leq k \leq n-1) \\
\text { (iii) }\left\langle Y^{n} X, Y^{k} X\right\rangle=\left\langle Y^{n+1}, Y^{k-1} X^{2}\right\rangle & (1 \leq k \leq n-1) \\
\text { (iv) }\left\langle Y^{n} X, Y^{k+1}\right\rangle=\left\langle Y^{n+1}, Y^{k} X\right\rangle & (0 \leq k \leq n-1) .
\end{array}\right.
$$

We first consider rows of $B$ corresponding to monomials of total degree at most $n-1$. To establish (2.9)(i) for $k \leq n-2$, we calculate

$$
\begin{aligned}
\left\langle Y^{n-1} X^{2}, Y^{k} X\right\rangle & =\left\langle Y^{n}, Y^{k} X\right\rangle=\left\langle Y^{n} X, Y^{k}\right\rangle \quad\left(\text { since } Y^{n-1} X^{2}=Y^{n}, \text { and by }(2.8)\right) \\
& =\left\langle Y^{n} X, Y^{k-1} X^{2}\right\rangle \quad(\text { by }(2.6)) .
\end{aligned}
$$

To verify (2.9)(ii) for $k \leq n-2$, we have

$$
\begin{aligned}
\left\langle Y^{n-1} X^{2}, Y^{k+1}\right\rangle & \left.=\left\langle Y^{n}, Y^{k+1}\right\rangle=\left\langle p_{n-1}(Y)+q_{n-1}(Y) X, Y^{k+1}\right\rangle \quad \text { by }(2.3)\right) \\
& =\left\langle p_{n-1}(Y), Y^{k+1}\right\rangle+\left\langle q_{n-1}(Y) X, Y^{k+1}\right\rangle \\
& =\left\langle Y^{k+1}, p_{n-1}(Y)\right\rangle+\left\langle q_{n-1}(Y) X, Y^{k+1}\right\rangle \quad \text { (by Lemma 2.9(i)) } \\
& =\left\langle Y^{k} X^{2}, p_{n-1}(Y)\right\rangle+\left\langle q_{n-1}(Y) X, Y^{k+1}\right\rangle \quad\left(\text { using } Y=X^{2}\right) \\
& =\left\langle p_{n-1}(Y), Y^{k} X^{2}\right\rangle+\left\langle q_{n-1}(Y) X, Y^{k+1}\right\rangle \quad \text { (by Lemma 2.9(i)) } \\
& =\left\langle p_{n-1}(Y) X, Y^{k} X\right\rangle+\left\langle q_{n-1}(Y), Y^{k+1} X\right\rangle \\
& =\left\langle p_{n-1}(Y) X, Y^{k} X\right\rangle+\left\langle q_{n-1}(Y) Y, Y^{k} X\right\rangle \\
& =\left\langle p_{n-1}(Y) X+q_{n-1}(Y) Y, Y^{k} X\right\rangle \\
& =\left\langle Y^{n} X, Y^{k} X\right\rangle \quad(\text { by }(2.4)) .
\end{aligned}
$$


Next, consider $(2.9)$ (iii) with $k \leq n-2$. Write $q_{n-1}(Y) \equiv r_{n-2}(Y)+c_{n-1} Y^{n-1}$, with $\operatorname{deg} r_{n-2} \leq n-2$; then

$$
\begin{aligned}
\left\langle Y^{n+1}, Y^{k-1} X^{2}\right\rangle & \left.=\left\langle p_{n-1}(Y) Y+q_{n-1}(Y) Y X, Y^{k-1} X^{2}\right\rangle \quad \text { by }(2.5)\right) \\
& =\left\langle p_{n-1}(Y), Y^{k} X^{2}\right\rangle+\left\langle\left[r_{n-2}(Y)+c_{n-1} Y^{n-1}\right] Y X, Y^{k}\right\rangle \quad \text { by (2.6)) } \\
& =\left\langle p_{n-1}(Y) X, Y^{k} X\right\rangle+\left\langle r_{n-2}(Y) Y, Y^{k} X\right\rangle+c_{n-1}\left\langle Y^{n} X, Y^{k}\right\rangle \\
& =\left\langle p_{n-1}(Y) X, Y^{k} X\right\rangle+\left\langle r_{n-2}(Y) Y, Y^{k} X\right\rangle+c_{n-1}\left\langle Y^{n}, Y^{k} X\right\rangle \\
& (\text { by }(2.8)) \\
& =\left\langle p_{n-1}(Y) X+\left[r_{n-2}(Y)+c_{n-1} Y^{n-1}\right] Y, Y^{k} X\right\rangle \\
& =\left\langle p_{n-1}(Y) X+q_{n-1}(Y) Y, Y^{k} X\right\rangle \\
& \left.=\left\langle Y^{n} X, Y^{k} X\right\rangle \quad \text { by }(2.4)\right) .
\end{aligned}
$$$$
=\left\langle p_{n-1}(Y) X, Y^{k} X\right\rangle+\left\langle r_{n-2}(Y) Y, Y^{k} X\right\rangle+c_{n-1}\left\langle Y^{n} X, Y^{k}\right\rangle
$$

Now we prove $(2.9)$ (iv) for $k \leq n-2$. We have

$$
\begin{aligned}
\left\langle Y^{n} X, Y^{k+1}\right\rangle & =\left\langle p_{n-1}(Y) X+q_{n-1}(Y) Y, Y^{k+1}\right\rangle \\
& =\left\langle p_{n-1}(Y) X, Y^{k+1}\right\rangle+\left\langle q_{n-1}(Y) Y, Y^{k+1}\right\rangle \\
& =\left\langle p_{n-1}(Y), Y^{k+1} X\right\rangle+\left\langle q_{n-1}(Y) Y, Y^{k+1}\right\rangle \\
& =\left\langle p_{n-1}(Y) Y, Y^{k} X\right\rangle+\left\langle q_{n-1}(Y), Y^{k+2}\right\rangle
\end{aligned}
$$

and

$$
\begin{aligned}
\left\langle Y^{n+1}, Y^{k} X\right\rangle & =\left\langle p_{n-1}(Y) Y+q_{n-1}(Y) Y X, Y^{k} X\right\rangle \\
& =\left\langle p_{n-1}(Y) Y, Y^{k} X\right\rangle+\left\langle q_{n-1}(Y) Y X, Y^{k} X\right\rangle .
\end{aligned}
$$

Thus, the Hankel condition $\left\langle Y^{n} X, Y^{k+1}\right\rangle=\left\langle Y^{n+1}, Y^{k} X\right\rangle$ is satisfied if and only if

$$
\left\langle Y^{j}, Y^{k+2}\right\rangle=\left\langle Y^{j} Y X, Y^{k} X\right\rangle(0 \leq j \leq n-1) \text {. }
$$

For $j \leq n-2$, we have

$$
\left\langle Y^{j}, Y^{k+2}\right\rangle=\left\langle Y^{j} X^{2}, Y^{k+1}\right\rangle=\left\langle Y^{j} X, Y^{k+1} X\right\rangle=\left\langle Y^{j} Y X, Y^{k} X\right\rangle,
$$

and for $j=n-1$,

$$
\begin{aligned}
\left\langle Y^{n-1}, Y^{k+2}\right\rangle & \left.=\left\langle Y^{n}, Y^{k+1}\right\rangle=\left\langle Y^{n-1} X^{2}, Y^{k+1}\right\rangle \quad \text { (by the definition of } Y^{n-1} X^{2}\right) \\
& =\left\langle Y^{n} X, Y^{k} X\right\rangle \quad \text { by }(2.9(\text { ii }) \text { for } k \leq n-2) \\
& =\left\langle Y^{n-1} Y X, Y^{k} X\right\rangle,
\end{aligned}
$$

as desired. 
We now consider the case of (2.9) when the rows have total degree $n$, i.e., $k=n-1$. To establish (2.9)(i) for $k=n-1$, we calculate

$$
\begin{aligned}
\left\langle Y^{n-1} X^{2}, Y^{n-1} X\right\rangle & \left.=\left\langle Y^{n}, Y^{n-1} X\right\rangle=\left\langle p_{n-1}(Y)+q_{n-1}(Y) X, Y^{n-1} X\right\rangle \quad \text { (by }(2.3)\right) \\
& =\left\langle p_{n-1}(Y) X, Y^{n-1}\right\rangle+\left\langle q_{n-1}(Y) X, Y^{n-1} X\right\rangle .
\end{aligned}
$$

We next verify that $\left\langle Y^{n-1} X, Y^{n-1} X\right\rangle=\left\langle Y^{n-1} X^{2}, Y^{n-1}\right\rangle$; indeed,

$$
\begin{aligned}
\left\langle Y^{n-1} X, Y^{n-1} X\right\rangle & =\left\langle Y^{n}, Y^{n-2} X^{2}\right\rangle \quad \text { (since } B_{n, n} \text { is Hankel) } \\
& =\left\langle Y^{n}, Y^{n-1}\right\rangle \quad \text { (by Lemma } 2.9(\text { iii }) \text { ) } \\
& \left.=\left\langle Y^{n-1} X^{2}, Y^{n-1}\right\rangle \quad \text { (by the definition of } Y^{n-1} X^{2}\right) .
\end{aligned}
$$

Now, the expression in (2.10) coincides with

$$
\begin{aligned}
& \left\langle p_{n-1}(Y) X, Y^{n-1}\right\rangle+\left\langle q_{n-1}(Y) X^{2}, Y^{n-1}\right\rangle \\
& =\left\langle p_{n-1}(Y) X+q_{n-1}(Y) Y, Y^{n-1}\right\rangle \quad\left(\text { since } Y^{n-1} X^{2}=Y^{n}\right) \\
& =\left\langle Y^{n} X, Y^{n-1}\right\rangle=\left\langle Y^{n} X, Y^{n-2} X^{2}\right\rangle \quad(\text { by }(2.6)) .
\end{aligned}
$$

For $(2.9)$ (ii) with $k=n-1$, note first that

$$
\begin{aligned}
\left\langle Y^{n-1} X^{2}, Y^{n}\right\rangle & \left.=\left\langle Y^{n}, Y^{n}\right\rangle=\left\langle p_{n-1}(Y)+q_{n-1}(Y) X, Y^{n}\right\rangle \quad \text { (by }(2.3)\right) \\
& =\left\langle p_{n-1}(Y), Y^{n}\right\rangle+\left\langle q_{n-1}(Y) X, Y^{n}\right\rangle .
\end{aligned}
$$

Next, we claim that

$$
\left\langle p_{n-1}(Y) X, Y^{n-1} X\right\rangle=\left\langle p_{n-1}(Y), Y^{n}\right\rangle
$$

Indeed, for $j \leq n-2,\left\langle Y^{j} X, Y^{n-1} X\right\rangle=\left\langle Y^{j} X^{2}, Y^{n-1}\right\rangle=\left\langle Y^{j} Y, Y^{n-1}\right\rangle=\left\langle Y^{j}, Y^{n}\right\rangle$, while $\left\langle Y^{n-1} X, Y^{n-1} X\right\rangle=$ $\left\langle Y^{n}, Y^{n-1}\right\rangle$ (as we have shown above, in the proof of (2.9) with $k=n-1$ ). It follows that

$$
\begin{aligned}
\left\langle Y^{n} X, Y^{n-1} X\right\rangle & =\left\langle p_{n-1}(Y) X+q_{n-1}(Y) Y, Y^{n-1} X\right\rangle \\
& =\left\langle p_{n-1}(Y) X, Y^{n-1} X\right\rangle+\left\langle q_{n-1}(Y) Y, Y^{n-1} X\right\rangle . \\
& =\left\langle p_{n-1}(Y), Y^{n}\right\rangle+\left\langle q_{n-1}(Y) Y, Y^{n-1} X\right\rangle \quad \text { (by (2.12)). }
\end{aligned}
$$

We thus see from (2.11) and (2.13) that

$$
\left\langle Y^{n-1} X^{2}, Y^{n}\right\rangle=\left\langle Y^{n} X, Y^{n-1} X\right\rangle
$$

if and only if $\left\langle q_{n-1}(Y) X, Y^{n}\right\rangle=\left\langle q_{n-1}(Y) Y, Y^{n-1} X\right\rangle$; this reduces to verifying that $\left\langle Y^{n-1} X, Y^{n}\right\rangle=$ $\left\langle Y^{n}, Y^{n-1} X\right\rangle$, which follows from the self-adjointness of $\mathcal{M}(n)$.

To verify (2.9)(iii) for $k=n-1$ we need to show that $\left\langle Y^{n} X, Y^{n-1} X\right\rangle=\left\langle Y^{n+1}, Y^{n-2} X^{2}\right\rangle$. Observe that

$$
\begin{aligned}
\left\langle Y^{n+1}, Y^{n-2} X^{2}\right\rangle & =\left\langle p_{n-1}(Y) Y+q_{n-1}(Y) Y X, Y^{n-2} X^{2}\right\rangle \quad(\text { by }(2.5)) \\
& =\left\langle p_{n-1}(Y) Y+q_{n-1}(Y) Y X, Y^{n-1}\right\rangle \quad(\text { by }(2.6))
\end{aligned}
$$

We claim that $\left\langle Y^{n-1} X, Y^{n}\right\rangle=\left\langle Y^{n} X, Y^{n-1}\right\rangle ;$ indeed,

$$
\begin{aligned}
\left\langle Y^{n-1} X, Y^{n}\right\rangle & =\left\langle Y^{n}, Y^{n-1} X\right\rangle=\left\langle Y^{n-1} X^{2}, Y^{n-1} X\right\rangle \\
& \text { (by the definition of } \left.Y^{n-1} X^{2}\right) \\
& =\left\langle Y^{n} X, Y^{n-2} X^{2}\right\rangle \quad(\text { by }(2.9)(\mathrm{i}) \text { with } k=n-1) \\
& =\left\langle Y^{n} X, Y^{n-1}\right\rangle \quad(\text { by }(2.6)) .
\end{aligned}
$$


Now, the expression in (2.15) coincides with

$$
\begin{aligned}
\left\langle p_{n-1}(Y) Y, Y^{n-1}\right\rangle+\left\langle q_{n-1}(Y) X, Y^{n}\right\rangle & =\left\langle p_{n-1}(Y)+q_{n-1}(Y) X, Y^{n}\right\rangle \\
& =\left\langle Y^{n}, Y^{n}\right\rangle=\left\langle Y^{n-1} X^{2}, Y^{n}\right\rangle \\
& =\left\langle Y^{n} X, Y^{n-1} X\right\rangle \text { (by (2.14)), }
\end{aligned}
$$

as desired. To complete the case $k=n-1$ we need to show condition (iv) in (2.9) holds, that is, $\left\langle Y^{n} X, Y^{n}\right\rangle=\left\langle Y^{n+1}, Y^{n-1} X\right\rangle$. We do this as follows:

$$
\begin{aligned}
\left\langle Y^{n} X, Y^{n}\right\rangle & =\left\langle p_{n-1}(Y) X+q_{n-1}(Y) Y, Y^{n}\right\rangle \quad \text { (by (2.4) } \\
& =\left\langle p_{n-1}(Y) X, Y^{n}\right\rangle+\left\langle q_{n-1}(Y) Y, Y^{n}\right\rangle
\end{aligned}
$$

and

$$
\begin{aligned}
\left\langle Y^{n+1}, Y^{n-1} X\right\rangle & =\left\langle p_{n-1}(Y) Y+q_{n-1}(Y) Y X, Y^{n-1} X\right\rangle \quad \text { (by }(2.5) \\
& =\left\langle p_{n-1}(Y) Y, Y^{n-1} X\right\rangle+\left\langle q_{n-1}(Y) Y X, Y^{n-1} X\right\rangle .
\end{aligned}
$$

It thus suffices to verify that

$$
\left\langle p_{n-1}(Y) X, Y^{n}\right\rangle=\left\langle p_{n-1}(Y) Y, Y^{n-1} X\right\rangle
$$

and

$$
\left\langle q_{n-1}(Y) Y, Y^{n}\right\rangle=\left\langle q_{n-1}(Y) Y X, Y^{n-1} X\right\rangle .
$$

The first equality follows from $\left\langle Y^{j} X, Y^{n}\right\rangle=\left\langle Y^{j+1}, Y^{n-1} X\right\rangle(0 \leq j \leq n-2)$ and from $\left\langle Y^{n-1} X, Y^{n}\right\rangle=$ $\left\langle Y^{n}, Y^{n-1} X\right\rangle$ (by the self-adjointness of $\mathcal{M}(n)$ ). To prove the second equality, note first that in $\mathcal{C}_{\mathcal{M}(n)}$,

$$
\left\langle Y^{j+1} X, Y^{n-1} X\right\rangle=\left\langle Y^{j+1} X^{2}, Y^{n-1}\right\rangle=\left\langle Y^{j+1} Y, Y^{n-1}\right\rangle=\left\langle Y^{j+1}, Y^{n}\right\rangle
$$

for $0 \leq j \leq n-3$. Further,

$$
\left\langle Y^{n-1}, Y^{n}\right\rangle=\left\langle Y^{n}, Y^{n-1}\right\rangle=\left\langle Y^{n}, Y^{n-2} X^{2}\right\rangle=\left\langle Y^{n-1} X, Y^{n-1} X\right\rangle
$$

(by the Hankel property in $\mathcal{M}(n)$ ). Finally,

$$
\begin{aligned}
\left\langle Y^{n} X, Y^{n-1} X\right\rangle & \left.=\left\langle Y^{n-1} X^{2}, Y^{n}\right\rangle \text { (by }(2.14)\right) \\
& \left.=\left\langle Y^{n}, Y^{n}\right\rangle \quad \text { (by the definition of } Y^{n-1} X^{2}\right) .
\end{aligned}
$$

The proof that block $B$ is of the form $\left\{B_{i, n+1}\right\}_{i=0}^{n}$ is now complete.

To finish the proof of Proposition 2.8 it now suffices to show that $C:=W^{*} \mathcal{M}(n) W$ is Hankel. Observe that in $\mathcal{M}:=[\mathcal{M}(n) \quad B]=\left(\begin{array}{cc}\mathcal{M}(n) & B \\ B^{*} & C\end{array}\right)$, we may compute inner products of the form $\langle p(X, Y), q(X, Y)\rangle$ where $p, q \in \mathcal{P}_{n+1}^{\mathbb{R}}$. Note also that since $\mathcal{M}$ is a flat extension, dependence relations in the columns of $(\mathcal{M}(n) B)$ extend to column relations in ( $B^{*} C$ ). In particular, the first $n$ columns of $C$ coincide with the last $n$ columns of $B^{*}$; since $B$ has the Hankel property, so does $B^{*}$, and thus the first $n$ columns of $C$ have the Hankel property. Further, columns $Y^{n} X$ and $Y^{n+1}$ of $C$ are defined as in (2.4) and (2.5), respectively. To verify that $C$ is Hankel it now suffices to focus on the last three columns of $C$, namely $Y^{n-1} X^{2}, Y^{n} X$ and $Y^{n+1}$. We will first compare the entries of $Y^{n-1} X^{2}$ and $Y^{n} X$, and later those of $Y^{n} X$ and $Y^{n+1}$. To this end, we need three preliminary facts.

Claim 1. For $0 \leq i \leq n-1$,

$$
\left\langle Y^{n-1} X^{2}, Y^{i} X^{n+1-i}\right\rangle=\left\langle Y^{n-1} X^{2}, Y^{i+1} X^{n-1-i}\right\rangle
$$




\section{Proof.}

$$
\left\langle Y^{n-1} X^{2}, Y^{i} X^{n+1-i}\right\rangle=\left\langle Y^{n}, Y^{i} X^{n+1-i}\right\rangle
$$

(by the definitions of the columns of $C$ )

$$
\begin{aligned}
& =\left\langle Y^{i} X^{n+1-i}, Y^{n}\right\rangle \quad\left(\text { since } \mathcal{M}=\mathcal{M}^{*}\right) \\
& =\left\langle Y^{i+1} X^{n-1-i}, Y^{n}\right\rangle
\end{aligned}
$$

(by the definition of the columns of $C$ )

$$
\begin{aligned}
& =\left\langle Y^{n}, Y^{i+1} X^{n-1-i}\right\rangle \quad\left(\text { since } \mathcal{M}=\mathcal{M}^{*}\right) \\
& =\left\langle Y^{n-1} X^{2}, Y^{i+1} X^{n-1-i}\right\rangle
\end{aligned}
$$

(by the definitions of the columns of $C$ ).

Claim 2. For $0 \leq i \leq n-1$,

$$
\left\langle Y^{n} X, Y^{i} X^{n+1-i}\right\rangle=\left\langle Y^{n} X, Y^{i+1} X^{n-1-i}\right\rangle
$$

Proof.

$$
\begin{aligned}
\left\langle Y^{n} X, Y^{i} X^{n+1-i}\right\rangle & =\left\langle p_{n-1}(Y) X+q_{n-1}(Y) Y, Y^{i} X^{n+1-i}\right\rangle \quad(\text { by }(2.4)) \\
& =\left\langle Y^{i} X^{n+1-i}, p_{n-1}(Y) X+q_{n-1}(Y) Y\right\rangle \quad\left(\text { since } \mathcal{M}=\mathcal{M}^{*}\right) \\
& =\left\langle Y^{i+1} X^{n-1-i}, p_{n-1}(Y) X+q_{n-1}(Y) Y\right\rangle
\end{aligned}
$$

(by the definition of the columns of $C$ )

$$
\begin{aligned}
& =\left\langle p_{n-1}(Y) X+q_{n-1}(Y) Y, Y^{i+1} X^{n-1-i}\right\rangle \quad\left(\text { since } \mathcal{M}=\mathcal{M}^{*}\right) \\
& =\left\langle Y^{n} X, Y^{i+1} X^{n-1-i}\right\rangle \quad(\text { by }(2.4)) .
\end{aligned}
$$

Claim 3. For $0 \leq i \leq n-1$,

$$
\left\langle Y^{n+1}, Y^{i} X^{n+1-i}\right\rangle=\left\langle Y^{n+1}, Y^{i+1} X^{n-1-i}\right\rangle
$$

\section{Proof.}

$$
\begin{aligned}
\left\langle Y^{n+1}, Y^{i} X^{n+1-i}\right\rangle & =\left\langle p_{n-1}(Y) Y+q_{n-1}(Y) Y X, Y^{i} X^{n+1-i}\right\rangle \quad(\text { by }(2.5)) \\
& =\left\langle Y^{i} X^{n+1-i}, p_{n-1}(Y) Y+q_{n-1}(Y) Y X\right\rangle \quad\left(\text { since } \mathcal{M}=\mathcal{M}^{*}\right) \\
& =\left\langle Y^{i+1} X^{n-1-i}, p_{n-1}(Y) Y+q_{n-1}(Y) Y X\right\rangle
\end{aligned}
$$

(by the definition of the columns of $C$ )

$$
\begin{aligned}
& =\left\langle p_{n-1}(Y) Y+q_{n-1}(Y) Y X, Y^{i+1} X^{n-1-i}\right\rangle \quad\left(\text { since } \mathcal{M}=\mathcal{M}^{*}\right) \\
& =\left\langle Y^{n+1}, Y^{i+1} X^{n-1-i}\right\rangle \quad(\text { by }(2.5)) .
\end{aligned}
$$

Comparison of $Y^{n-1} X^{2}$ and $Y^{n} X$. We will establish that

$$
\left\langle Y^{n-1} X^{2}, Y^{i} X^{n+1-i}\right\rangle=\left\langle Y^{n} X, Y^{i-1} X^{n+2-i}\right\rangle \quad(1 \leq i \leq n+1) \text {. }
$$

Case 1. $(1 \leq i \leq n-1)$ :

$$
\begin{aligned}
\left\langle Y^{n-1} X^{2}, Y^{i} X^{n+1-i}\right\rangle & =\left\langle Y^{n-1} X^{2}, Y^{i+1} X^{n-1-i}\right\rangle \quad \text { (by (2.18)) } \\
& =\left\langle Y^{n} X, Y^{i} X^{n-i}\right\rangle \quad \text { (because } B \text { is Hankel) } \\
& =\left\langle Y^{n} X, Y^{i-1} X^{n+2-i}\right\rangle \quad \text { (by (2.19)). }
\end{aligned}
$$

Case 2. $(i=n)$ This is straightforward from the self-adjointness of $C$. 
Case 3. $(i=n+1)$ We need to prove that

$$
\left\langle Y^{n-1} X^{2}, Y^{n+1}\right\rangle=\left\langle Y^{n} X, Y^{n} X\right\rangle
$$

Observe that

$$
\begin{aligned}
\left\langle Y^{n-1} X^{2}, Y^{n+1}\right\rangle & \left.=\left\langle Y^{n}, Y^{n+1}\right\rangle \quad \text { (by the definition of the columns of } C\right) \\
& =\left\langle Y^{n+1}, Y^{n}\right\rangle \quad\left(\text { since } \mathcal{M}=\mathcal{M}^{*}\right) \\
& =\left\langle p_{n-1}(Y) Y+q_{n-1}(Y) Y X, Y^{n}\right\rangle \quad(\text { by }(2.5))
\end{aligned}
$$

Similarly,

$$
\left\langle Y^{n} X, Y^{n} X\right\rangle=\left\langle p_{n-1}(Y) X+q_{n-1}(Y) Y, Y^{n} X\right\rangle .
$$

It follows that to verify (2.21) it suffices to establish

$$
\left\langle p_{n-1}(Y) Y, Y^{n}\right\rangle=\left\langle p_{n-1}(Y) X, Y^{n} X\right\rangle
$$

and

$$
\left\langle q_{n-1}(Y) Y X, Y^{n}\right\rangle=\left\langle q_{n-1}(Y) Y, Y^{n} X\right\rangle .
$$

For (2.22), we will verify that for $0 \leq j \leq n-1$,

$$
\left\langle Y^{j+1}, Y^{n}\right\rangle=\left\langle Y^{j} X, Y^{n} X\right\rangle .
$$

We have

$$
\left\langle Y^{j+1}, Y^{n}\right\rangle=\left\langle Y^{n}, Y^{j+1}\right\rangle=\left\langle p_{n-1}(Y)+q_{n-1}(Y) X, Y^{j+1}\right\rangle
$$

and

$$
\left\langle Y^{j} X, Y^{n} X\right\rangle=\left\langle Y^{n} X, Y^{j} X\right\rangle=\left\langle p_{n-1}(Y) X+q_{n-1}(Y) Y, Y^{j} X\right\rangle .
$$

To establish (2.24), it now suffices to prove that

$$
\left\langle Y^{k}, Y^{j+1}\right\rangle=\left\langle Y^{k} X, Y^{j} X\right\rangle \quad(0 \leq k \leq n-1,0 \leq j \leq n-1)
$$

and

$$
\left\langle Y^{k} X, Y^{j+1}\right\rangle=\left\langle Y^{k+1}, Y^{j} X\right\rangle \quad(0 \leq k \leq n-1,0 \leq j \leq n-1) .
$$

For (2.25), we have

$$
\begin{aligned}
\left\langle Y^{k} X, Y^{j} X\right\rangle & =\left\langle Y^{j} X, Y^{k} X\right\rangle=\left\langle Y^{j+1}, Y^{k-1} X^{2}\right\rangle \\
& \text { (by the Hankel property in } \mathcal{M}(n)) \\
& \left.=\left\langle Y^{j+1}, Y^{k}\right\rangle \quad \text { (via recursiveness and self-adjointness in } \mathcal{M}(n)\right) \\
& =\left\langle Y^{k}, Y^{j+1}\right\rangle .
\end{aligned}
$$

For (2.26), first consider the case when $j, k \leq n-2$; then

$$
\begin{aligned}
\left\langle Y^{k} X, Y^{j+1}\right\rangle & =\left\langle Y^{k} X, Y^{j} X^{2}\right\rangle \\
& =\left\langle Y^{k} X^{2}, Y^{j} X\right\rangle=\left\langle Y^{k+1}, Y^{j} X\right\rangle .
\end{aligned}
$$

Now consider (2.26) with $j=n-1, k \leq n-1$; we have

$$
\begin{aligned}
\left\langle Y^{k+1}, Y^{n-1} X\right\rangle & =\left\langle Y^{n-1} X, Y^{k+1}\right\rangle=\left\langle Y^{n}, Y^{k} X\right\rangle \\
& \text { (by the Hankel property in } \mathcal{M}(n)) \\
& =\left\langle Y^{k} X, Y^{n}\right\rangle .
\end{aligned}
$$


Finally, for (2.26) with $k=n-1, j \leq n-1$, note that $\left\langle Y^{n-1} X, Y^{j+1}\right\rangle=\left\langle Y^{n}, Y^{j} X\right\rangle$, by the Hankel property in $\mathcal{M}(n)$. Thus, we have established (2.26), whence (2.22) follows. We next prove (2.23); to do so, it suffices to establish that

$$
\left\langle Y^{j+1} X, Y^{n}\right\rangle=\left\langle Y^{j+1}, Y^{n} X\right\rangle \quad(0 \leq j \leq n-1) .
$$

Consider first the case when $j \leq n-3$; then

$$
\begin{aligned}
\left\langle Y^{j+1}, Y^{n} X\right\rangle & =\left\langle Y^{n} X, Y^{j+1}\right\rangle \\
& =\left\langle Y^{n}, Y^{j+1} X\right\rangle \quad(\text { by }(2.8)) \\
& =\left\langle Y^{j+1} X, Y^{n}\right\rangle .
\end{aligned}
$$

For (2.27) with $j=n-2$, we have

$$
\begin{aligned}
\left\langle Y^{n-1}, Y^{n} X\right\rangle & =\left\langle Y^{n} X, Y^{n-1}\right\rangle=\left\langle p_{n-1}(Y) X+q_{n-1}(Y) Y, Y^{n-1}\right\rangle \\
& =\left\langle p_{n-1}(Y) X, Y^{n-1}\right\rangle+\left\langle q_{n-1}(Y) Y, Y^{n-1}\right\rangle \\
& =\left\langle p_{n-1}(Y), Y^{n-1} X\right\rangle+\left\langle q_{n-1}(Y) X, Y^{n-1} X\right\rangle \quad \text { (using (2.16)) } \\
& =\left\langle p_{n-1}(Y)+q_{n-1}(Y) X, Y^{n-1} X\right\rangle \\
& =\left\langle Y^{n}, Y^{n-1} X\right\rangle=\left\langle Y^{n-1} X, Y^{n}\right\rangle .
\end{aligned}
$$

Finally, (2.27) with $j=n-1$ follows from self-adjointness in $\mathcal{M}:\left\langle Y^{n} X, Y^{n}\right\rangle=\left\langle Y^{n}, Y^{n} X\right\rangle$. This concludes the proof of (2.27); thus (2.23) is established and the proof of Case 3 is complete.

Comparison of $Y^{n} X$ and $Y^{n+1}$. We will establish that $\left\langle Y^{n} X, Y^{i} X^{n+1-i}\right\rangle=\left\langle Y^{n+1}, Y^{i-1} X^{n+2-i}\right\rangle$ $(1 \leq i \leq n+1)$.

Case $1(1 \leq i \leq n-1)$ :

$$
\begin{aligned}
\left\langle Y^{n} X, Y^{i} X^{n+1-i}\right\rangle & =\left\langle Y^{n} X, Y^{i+1} X^{n-1-i}\right\rangle \quad(\text { by }(2.19)) \\
& =\left\langle Y^{n+1}, Y^{i} X^{n-i}\right\rangle \quad(\text { by the Hankel property in } B) \\
& =\left\langle Y^{n+1}, Y^{i-1} X^{n+2-i}\right\rangle \quad(\text { by }(2.20)) .
\end{aligned}
$$

Case 2. $(i=n)$ This is $(2.21)$.

Case 3. $(i=n+1)$ This is straightforward from the self-adjointness of $C$.

This concludes the proof of Proposition 2.8.

Proposition 2.13. (Case $V: \operatorname{rank} \mathcal{M}(n)=2 n+1$ ) Assume that $\mathcal{M}(n)$ is positive, recursively

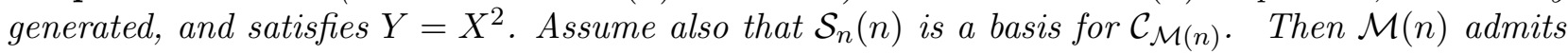
a one-parameter family of flat extensions $\mathcal{M}(n+1)$.

Proof. Since $Y=X^{2}$, and to guarantee that $\mathcal{M}(n+1)$ is recursively generated, we define the first $n$ columns of a proposed $B$ block for $\mathcal{M}(n+1)$ as $\left[X^{n+1}\right]_{m(n)}:=Y X^{n-1} \in \mathcal{C}_{\mathcal{M}(n)},\left[Y X^{n}\right]_{m(n)}:=$ $Y^{2} X^{n-2} \in \mathcal{C}_{\mathcal{M}(n)}, \ldots,\left[Y^{n-1} X^{2}\right]_{m(n)}:=Y^{n} \in \mathcal{C}_{\mathcal{M}(n)}$. Moreover, if we wish to make $B_{n, n+1}$ Hankel, it is clear that all but the last entry in the column $\left[Y^{n} X\right]_{m(n)}$ must be given in terms of the entries in $\mathcal{M}(n)$, and that all but the last entry in $\left[Y^{n+1}\right]_{m(n)}$ must be given in terms of the entries in $\left[Y^{n} X\right]_{m(n)} ;$ concretely,

$$
\left\langle Y^{n} X, Y^{k}\right\rangle:=\beta_{n+k, 1} \quad(0 \leq k \leq n-1)
$$


and

$$
\begin{aligned}
& \left\langle Y^{n} X, Y^{i+1} X^{j}\right\rangle:=\left\langle Y^{n-1} X^{2}, Y^{i+1} X^{j-1}\right\rangle= \\
& \left\langle Y^{n}, Y^{i+1} X^{j-1}\right\rangle \equiv \beta_{n+i+1, j-1}(i \geq 0, j \geq 1, i+j \leq n) .
\end{aligned}
$$

To handle the last entry of $\left[Y^{n} X\right]_{m(n)}$, we introduce the parameter $p \equiv\left\langle Y^{n} X, Y^{n}\right\rangle_{\mathcal{M}(n+1)}$. Similarly, we let

$$
\begin{gathered}
\left\langle Y^{n+1}, Y^{k}\right\rangle:=\beta_{n+1+k, 0}(0 \leq k \leq n-1), \\
\left\langle Y^{n+1}, Y^{i} X^{j}\right\rangle:=\left\langle Y^{n} X, Y^{i+1} X^{j-1}\right\rangle \quad(i \geq 0, j \geq 1, i+j \leq n),
\end{gathered}
$$

and $q:=\left\langle Y^{n+1}, Y^{n}\right\rangle$.

Claim 1.

$$
\left\langle Y^{n} X, Y^{i} X^{j+2}\right\rangle=\left\langle Y^{n} X, Y^{i+1} X^{j}\right\rangle(i+j+2 \leq n) .
$$

Proof. Assume first $j \geq 1$. Then

$$
\begin{aligned}
\left\langle Y^{n} X, Y^{i} X^{j+2}\right\rangle & =\left\langle Y^{n}, Y^{i+1} X^{j+1}\right\rangle \\
& =\left\langle Y^{n}, Y^{i+2} X^{j-1}\right\rangle \quad\left(\text { since } Y=X^{2}\right) \\
& =\left\langle Y^{n} X, Y^{i+1} X^{j}\right\rangle .
\end{aligned}
$$

If $j=0$,

$$
\left\langle Y^{n} X, Y^{i} X^{2}\right\rangle=\left\langle Y^{n}, Y^{i+1} X\right\rangle=\beta_{n+i+1,1}=\left\langle Y^{n} X, Y^{i+1}\right\rangle .
$$

Claim 2.

$$
\left\langle Y^{n+1}, Y^{i} X^{j+2}\right\rangle=\left\langle Y^{n+1}, Y^{i+1} X^{j}\right\rangle \quad(i+j+2 \leq n) .
$$

Proof. Assume first $j \geq 1$. Then

$$
\begin{aligned}
\left\langle Y^{n+1}, Y^{i} X^{j+2}\right\rangle & =\left\langle Y^{n} X, Y^{i+1} X^{j+1}\right\rangle \\
& =\left\langle Y^{n} X, Y^{i+2} X^{j-1}\right\rangle \quad \text { (by Claim 1) } \\
& =\left\langle Y^{n+1}, Y^{i+1} X^{j}\right\rangle .
\end{aligned}
$$

If $j=0$,

$$
\left\langle Y^{n+1}, Y^{i} X^{2}\right\rangle=\left\langle Y^{n} X, Y^{i+1} X\right\rangle=\left\langle Y^{n}, Y^{i+2}\right\rangle=\beta_{n+i+2,0}=\left\langle Y^{n+1}, Y^{i+1}\right\rangle .
$$

Repeated application of Claims 1 and 2 show that the each row of $B$ is identical to a row whose associated monomial corresponds to a column in the basis $\mathcal{S}_{n}(n)$, a property clearly present in $\mathcal{M}(n)$. This will be crucial in establishing that both $\left[Y^{n} X\right]_{m(n)}$ and $\left[Y^{n+1}\right]_{m(n)}$ are in the range of $\mathcal{M}(n)$.

Since $N:=\mathcal{M}(n)_{\mathcal{S}(n)}>0$, there exist vectors $\mathbf{f}, \mathbf{g} \in \mathbb{R}^{2 n+1}$ such that $N \mathbf{f}=\left[Y^{n} X\right]_{\mathcal{S}_{n}(n)}$ and $N \mathbf{g}=\left[Y^{n+1}\right]_{\mathcal{S}_{n}(n)}$. Let $\mathbf{F}, \mathbf{G} \in \mathbb{R}^{m(n)}$ be given by

$$
\left\langle\mathbf{F}, Y^{i} X^{j}\right\rangle:=\left\{\begin{array}{cl}
\left\langle\mathbf{f}, Y^{i} X^{j}\right\rangle & \text { if } Y^{i} X^{j} \in \mathcal{S}_{n}(n) \\
0 & \text { otherwise }
\end{array}\right.
$$

and

$$
\left\langle\mathbf{G}, Y^{i} X^{j}\right\rangle:=\left\{\begin{array}{cl}
\left\langle\mathbf{g}, Y^{i} X^{j}\right\rangle & \text { if } Y^{i} X^{j} \in \mathcal{S}_{n}(n) \\
0 & \text { otherwise }
\end{array} .\right.
$$


We claim that $\mathcal{M}(n) \mathbf{F}=\left[Y^{n} X\right]_{m(n)}$. Indeed, for $Y^{i} X^{j} \in \mathcal{S}_{n}(n)$, we have

$$
\begin{aligned}
\left\langle\mathcal{M}(n) \mathbf{F}, Y^{i} X^{j}\right\rangle & =\sum_{Y^{k} X^{\ell} \in \mathcal{C}_{\mathcal{M}(n)}}\left\langle Y^{k} X^{\ell}, Y^{i} X^{j}\right\rangle\left\langle\mathbf{F}, Y^{k} X^{\ell}\right\rangle \\
& =\sum_{Y^{k} X^{\ell} \in \mathcal{S}_{n}(n)}\left\langle Y^{k} X^{\ell}, Y^{i} X^{j}\right\rangle\left\langle\mathbf{f}, Y^{k} X^{\ell}\right\rangle \\
& +\sum_{Y^{k} X^{\ell} \notin \mathcal{S}_{n}(n)}\left\langle Y^{k} X^{\ell}, Y^{i} X^{j}\right\rangle \cdot 0 \\
& =\left\langle\left[Y^{n} X\right]_{\mathcal{S}_{n}(n)}, Y^{i} X^{j}\right\rangle=\left\langle\left[Y^{n} X\right]_{m(n)}, Y^{i} X^{j}\right\rangle .
\end{aligned}
$$

Further, for $Y^{i} X^{j} \in \mathcal{C}_{\mathcal{M}(n)} \backslash \mathcal{S}_{n}(n)$, there exist $i^{\prime}, j^{\prime}$ such that $Y^{i} X^{j}=Y^{i^{\prime}} X^{j^{\prime}} \in \mathcal{C}_{\mathcal{M}(n)}$ and $Y^{i^{\prime}} X^{j^{\prime}} \in$ $\mathcal{S}_{n}(n)$. Since $\mathcal{M}(n)$ is self-adjoint, row $Y^{i} X^{j}$ of $\mathcal{M}(n)$ coincides with row $Y^{i^{\prime}} X^{j^{\prime}}$. Now,

$$
\begin{aligned}
\left\langle\mathcal{M}(n) \mathbf{F}, Y^{i} X^{j}\right\rangle & =\sum_{Y^{k} X^{\ell} \in \mathcal{C}_{\mathcal{M}(n)}}\left\langle Y^{k} X^{\ell}, Y^{i} X^{j}\right\rangle\left\langle\mathbf{F}, Y^{k} X^{\ell}\right\rangle \\
& =\sum_{Y^{k} X^{\ell} \in \mathcal{C}_{\mathcal{M}(n)}}\left\langle Y^{k} X^{\ell}, Y^{i^{\prime}} X^{j^{\prime}}\right\rangle\left\langle\mathbf{F}, Y^{k} X^{\ell}\right\rangle \\
& =\left\langle\mathcal{M}(n) \mathbf{F}, Y^{i^{\prime}} X^{j^{\prime}}\right\rangle \\
& =\left\langle\left[Y^{n} X\right]_{m(n)}, Y^{i^{\prime}} X^{j^{\prime}}\right\rangle \quad \text { (from the preceding case) } \\
& =\left\langle\left[Y^{n} X\right]_{m(n)}, Y^{i} X^{j}\right\rangle \quad \text { (by Claim 1). }
\end{aligned}
$$

Thus $\mathcal{M}(n) \mathbf{F}=\left[Y^{n} X\right]_{m(n)}$, and a similar argument (using Claim 2) shows that $\mathcal{M}(n) \mathbf{G}=$ $\left[Y^{n+1}\right]_{m(n)}$. Since $\left[Y^{i} X^{j}\right]_{m(n)}=Y^{i+1} X^{j-2} \in \mathcal{C}_{\mathcal{M}(n)}$ for $i+j=n+1, j \geq 2$, it follows that $\operatorname{Ran} B \subseteq \operatorname{Ran} \mathcal{M}(n)$; thus there exists $W$ such that $\mathcal{M}(n) W=B$.

To show that the flat extension $\mathcal{M} \equiv[\mathcal{M}(n) ; B]$ is of the form $\mathcal{M}(n+1)$, it now suffices to show that $C:=W^{*} \mathcal{M}(n) W$ is Hankel. We have

$$
\mathcal{M}=\left(\begin{array}{cc}
\mathcal{M}(n) & B \\
B^{*} & C
\end{array}\right)
$$

recall that dependence relations in $\left(\begin{array}{ll}\mathcal{M}(n) & B\end{array}\right)$ extend to corresponding relations in $\left(\begin{array}{ll}B^{*} & C\end{array}\right)$. Now $B^{*}=\left(\begin{array}{ll}* & B_{n, n+1}^{*}\end{array}\right)$, where

$$
B_{n, n+1}^{*}=\left(\begin{array}{ccccc}
* & \cdots & * & * & * \\
\vdots & \ddots & \vdots & \vdots & \vdots \\
* & \cdots & * & * & * \\
* & \cdots & * & * & p \\
* & \cdots & * & p & q
\end{array}\right)_{(n+2) \times(n+1)}
$$

Since, in the column space of $(\mathcal{M}(n) \quad B)$, we have $X^{n+1}=Y X^{n-1}, \ldots$, $Y^{n-1} X^{2}=Y^{n}$, it follows that $C$ is of the form $C=\left(\tilde{B}_{n, n+1}^{*} *\right)_{(n+2) \times(n+2)}$, where $\tilde{B}_{n, n+1}^{*}$ is obtained from $B_{n, n+1}^{*}$ by deleting its leftmost column. Thus, since $\tilde{B}_{n, n+1}^{*}$ is Hankel and $C=C^{*}$, 
we have

$$
C=\left(\begin{array}{cccccc}
* & \cdots & * & * & * & * \\
\vdots & \ddots & \vdots & \vdots & \vdots & \vdots \\
* & \cdots & * & * & * & p \\
* & \cdots & * & * & p & q \\
* & \cdots & * & p & C_{n+1, n+1} & u \\
* & \cdots & p & q & u & v
\end{array}\right)
$$

for some $u, v \in \mathbb{R}$, and where each cross-diagonal that is not shown is constant. Observe that by the flat extension construction, $C_{n+1, n+1}=\left[Y^{n} X\right]_{m(n)}^{t} \mathbf{F}$. Since $\left[Y^{n} X\right]_{m(n)}$ is independent of $q$, so is $\mathbf{f}=N^{-1}\left[Y^{n} X\right]_{\mathcal{S}_{n}(n)}$, whence $\mathbf{F}$ is also independent of $q$. Thus $C_{n+1, n+1}$ does not depend on $q$ (though it does depend on $p$ ). For each $p$, if we let $q:=C_{n+1, n+1}(p)$, it follows that $\mathcal{M}=[\mathcal{M}(n) ; B]$ is a flat moment matrix extension of the form $\mathcal{M}(n+1)$. The proof is now complete.

\section{Proof of TheOREM 2.2}

We now turn to the proof of Theorem 2.2, which we restate for the sake of convenience; note that Theorem 1.4 follows from Theorem $3.1(i) \Leftrightarrow(v i)$.

Theorem 3.1. Let $\beta \equiv \beta^{(2 n)}: \beta_{00}, \beta_{01}, \beta_{10}, \ldots, \beta_{0,2 n}, \ldots, \beta_{2 n, 0}$ be a family of real numbers, $\beta_{00}>0$, and let $\mathcal{M}(n)$ be the associated moment matrix. Assume that $\mathcal{M}(n)$ is positive, recursively generated, and satisfies $Y=X^{2}$. The following statements are equivalent.

(i) $\beta$ admits a representing measure (necessarily supported in $y=x^{2}$ ).

(ii) $\beta$ admits a representing measure with moments of order up to $2 n+2$ (necessarily supported in $\left.y=x^{2}\right)$.

(iii) $\beta$ admits a rank $\mathcal{M}(n)$-atomic representing measure (necessarily supported in $y=x^{2}$ ).

(iv) $\mathcal{M}(n)$ admits a positive, recursively generated extension $\mathcal{M}(n+1)$.

(v) $\mathcal{M}(n)$ admits a flat extension $\mathcal{M}(n+1)$.

(vi) $\operatorname{rank} \mathcal{M}(n) \leq \operatorname{card} \mathcal{V}(\beta)$.

Proof. By [CuFi2, Theorem 2.1] and the equivalence of the moment problems for $M(n)(\gamma)$ and $\mathcal{M}(n)(\beta)$ [CuFi6, Proposition 1.12], we can assume that the columns 1, $X$ and $Y$ are linearly independent. Observe first that $(i i i) \Rightarrow(i i) \Rightarrow(i)$ trivially, that $(i) \Rightarrow(v i)$ by $[\mathrm{CuFi} 3,(1.7)]$, and that $(i i i) \Leftrightarrow(v)$ by [CuFi1, Theorem 5.13]. Also, $(v i) \Rightarrow(v)$ by Theorem 1.4 , and $(v) \Rightarrow(i v)$ by $[\mathrm{CuFi2}$, Lemma 1.9]. Thus, to finish the proof, it suffices to establish $(i v) \Rightarrow(v)$. We do this by considering the five cases in the proof of Theorem 1.4. First recall our hypotheses: $\mathcal{M}(n)$ is positive, recursively generated, $Y=X^{2}$, and $\mathcal{M}(n)$ admits a positive recursively generated extension $\mathcal{M}(n+1)$. We need to show that $\mathcal{M}(n)$ admits a flat extension.

Case I. The hypothesis about $\mathcal{M}(n+1)$ is superfluous, as we showed in Proposition 2.5 that $\mathcal{M}(n)$ is already flat, so it obviously admits a flat extension.

Case II. As above, the extra condition on $\mathcal{M}(n+1)$ is superfluous, since we proved in Proposition 2.6 that $\mathcal{M}(n)$ is flat.

Case III. Here we have

$$
Y^{n-1} X=p_{n-1}(Y)+q_{n-2}(Y) X
$$

in $\mathcal{C}_{\mathcal{M}(n)}$, with $\operatorname{deg} p_{n-1} \leq n-1$ and $\operatorname{deg} q_{n-2} \leq n-2$. By the Extension Principle [Fia1], the same relation must hold in the column space of the positive extension $\mathcal{M}(n+1)$. Since $\mathcal{M}(n+1)$ is recursively generated, we must also have

$$
Y^{n} \equiv Y^{n-1} X^{2}=Y^{n-1} X X=p_{n-1}(Y) X+q_{n-2}(Y) X X=p_{n-1}(Y) X+q_{n-2}(Y) Y \text {. }
$$


By (3.1), the first term in the last expression of (3.2) has total degree at most $n-1$, and so does the second term. It follows that $Y^{n}$ can be written in terms of columns of total degree at most $n-1$. Since $Y=X^{2}$ and $\mathcal{M}(n)$ is recursively generated, each column $Y^{i} X^{j}$ with $i+j=n$ and $j \geq 2$ coincides with a column of total degree at most $n-1$. It now follows that $\mathcal{M}(n)$ is flat, and thus admits a flat extension.

Case IV. Observe that since $\mathcal{M}(n+1)$ is recursively generated, we must have $X^{n+1}=Y X^{n-1}$, $Y X^{n}=Y^{2} X^{n-2}, \ldots, Y^{n-1} / X^{2}=Y^{n}$. By assumption, $Y^{n}$ is a linear combination of columns in $\mathcal{M}(n)$ of total degree at most $n-1$. By the Extension Principle [Fia1], the same relation holds in $\mathcal{M}(n+1)$. Since $\mathcal{M}(n+1)$ is recursively generated, we infer that $Y^{n} X$ and $Y^{n+1}$ are linear combinations of columns of degree at most $n$. Moreover, since $\mathcal{M}(n+1)$ is recursively generated and $Y=X^{2}$, the columns $Y^{i} X^{j}$ with $i+j=n+1$ and $j \geq 2$ coincide with columns of $\mathcal{M}(n+1)$ of total degree at most $n$. Thus $\mathcal{M}(n+1)$ is a flat extension of $\mathcal{M}(n)$.

Case V. Once again, we can ignore the given extension $\mathcal{M}(n+1)$ and obtain a flat extension from Proposition 2.13.

\section{Some Examples Illustrating Theorem 2.1}

Example 1.6 illustrates Case III of Theorem 2.1. We now present examples corresponding to the remaining cases of Theorem 2.1 .

Example 4.1. (Theorem 2.1, Cases IV and II) We define $\mathcal{M}(2)$ by

$$
\mathcal{M}(2):=\left(\begin{array}{cccccc}
1 & 0 & a & a & 0 & b \\
0 & a & 0 & 0 & b & 0 \\
a & 0 & b & b & 0 & c \\
a & 0 & b & b & 0 & c \\
0 & b & 0 & 0 & c & 0 \\
b & 0 & c & c & 0 & d
\end{array}\right),
$$

where $a>0, b>a^{2}, c>\frac{b^{2}}{a}$, and $d:=\frac{b^{3}-2 a b c+c^{2}}{b-a^{2}}$. Let $\sigma:=\frac{b^{2}-a c}{b-a^{2}}$ and $\tau:=\frac{c-a b}{b-a^{2}}$. Then $\mathcal{M}(2) \geq 0,\{1, X, Y, Y X\}$ is a basis for $\mathcal{C}_{\mathcal{M}(2)}, Y=X^{2}$, and $Y^{2}=\sigma 1+\tau Y$. Following the proof of Case IV, we define $B(3)$ via column relations $X^{3}:=Y X, Y X^{2}:=Y^{2}, Y^{2} X:=\sigma X+\tau Y X$, and $Y^{3}:=\sigma Y+\tau Y^{2}=\sigma \tau 1+\left(\sigma+\tau^{2}\right) Y$. A calculation shows that $[\mathcal{M}(2) ; B(3)]$ is indeed of the form $\mathcal{M}(3)$. To compute the corresponding 4-atomic representing measure, consider the equation $p(y):=y^{2}-\tau y-\sigma=0$. Since $\operatorname{card} \mathcal{V}\left(\beta^{(4)}\right) \geq \operatorname{rank} \mathcal{M}(2)=4, p$ must have two distinct positive roots, say $y_{1}$ and $y_{2}$. Explicitly, let $\omega:=\left(b-a^{2}\right)^{2}\left(\tau^{2}+4 \sigma\right) \equiv(c-a b)^{2}-4\left(b-a^{2}\right)\left(a c-b^{2}\right)$. Note that $\omega>0$ since

$$
a^{2} \omega \equiv\left[a^{2}\left(b-a^{2}\right)-a\left(c-\frac{b^{2}}{a}\right)\right]^{2}+\left(b-a^{2}\right)^{2}\left[2 a^{2}\left(b-a^{2}\right)+\left(b-a^{2}\right)^{2}+2 a\left(c-\frac{b^{2}}{a}\right)\right],
$$

so $y_{1}:=\frac{c-a b+\sqrt{\omega}}{2\left(b-a^{2}\right)}(>0)$ and $y_{2}:=\frac{c-a b-\sqrt{\omega}}{2\left(b-a^{2}\right)}(>0)$. Then $\mathcal{V}\left(\beta^{(4)}\right)=\left\{z_{1}, z_{2}, z_{3}, z_{4}\right\}$, where $z_{1}=$ $\left(\sqrt{y_{1}}, y_{1}\right), z_{2}=\left(-\sqrt{y_{1}}, y_{1}\right), z_{3}=\left(\sqrt{y_{2}}, y_{2}\right)$ and $z_{4}=\left(-\sqrt{y_{2}}, y_{2}\right)$. The unique 4 -atomic representing measure is $\mu:=\rho_{1} \delta_{z_{1}}+\rho_{2} \delta_{z_{2}}+\rho_{3} \delta_{z_{3}}+\rho_{4} \delta_{z_{4}}$, where $\rho_{1}=\rho_{2}=\frac{\omega-\left(2 a^{3}-3 a b+c\right) \sqrt{\omega}}{4 \omega}$ and $\rho_{3}=\rho_{4}=$ $\frac{\omega+\left(2 a^{3}-3 a b+c\right) \sqrt{\omega}}{4 \omega}$.

For a numerical example, let $a=2, b=5, c=13$; then $y_{1}=\frac{1}{2}(3+\sqrt{5}), y_{2}=\frac{1}{2}(3-\sqrt{5})$, $\rho_{1}=\rho_{2}=\frac{1}{20}(5+\sqrt{5}) \cong 0.361803, \rho_{3}=\rho_{4}=\frac{1}{20}(5-\sqrt{5}) \cong 0.138197$. To illustrate Case II, consider $\mathcal{M}(3)$; the first dependence relation in $\mathcal{S}_{3}(3)$ occurs at $Y^{k}$, with $2=k<n=3$. 
Example 4.2. (Theorem 2.1, Cases V and I) In Example 4.1, if we choose $d>\frac{b^{3}-2 a b c+c^{2}}{b-a^{2}}$, then $\mathcal{S}_{2}(2)$ is a basis for $\mathcal{C}_{\mathcal{M}(2)}$, so $\mathcal{M}(2)$ illustrates Case V. Following the proof of Case V, we define $Y^{2} X$ and $Y^{3}$ for block $B(3)$ by $Y^{2} X:=(0, c, 0,0, d, p)^{t}$ and $Y^{3}:=(c, 0, d, d, p, q)^{t}$, where $p, q \in \mathbb{R}$. Let $\lambda:=b^{3}-2 a b c+c^{2}+a^{2} d-b d(>0)$, and let $f_{1}:=-\left(a c-b^{2}\right) p / \lambda, f_{2}:=\left(c^{2}-b d\right) /\left(a c-b^{2}\right)$, $f_{3}:=(a b-c) p / \lambda, f_{4}:=0, f_{5}:=(a d-b c) /\left(a c-b^{2}\right), f_{6}:=-\left(b-a^{2}\right) p / \lambda$, and $\mathbf{F}:=\left(f_{1}, f_{2}, f_{3}, f_{4}, f_{5}, f_{6}\right)^{t}$. Then $Y^{2} X=\mathcal{M}(2) \mathbf{F}=f_{1} 1+f_{2} X+f_{3} Y+f_{5} Y X+f_{6} Y^{2}$, and a flat extension $\mathcal{M}(3)=[\mathcal{M}(2) ; B(3)]$ is uniquely determined by setting $q:=\left(Y^{2} X\right)^{t} \mathbf{F}=f_{2} c+f_{5} d+f_{6} p$. To determine the corresponding 5 -atomic representing measure $\mu_{p}$, observe that $5 \geq \operatorname{card}\left\{x: x^{5}=f_{1}+f_{2} x+f_{3} x^{2}+f_{5} x^{3}+f_{6} x^{4}\right\}=$ $\operatorname{card}\left\{(x, y): y=x^{2}\right.$ and $\left.x y^{2}=f_{1}+f_{2} x+f_{3} y+f_{5} x y+f_{6} y^{2}\right\} \geq \operatorname{card} \mathcal{V}(\beta) \geq \operatorname{rank} \mathcal{M}(2)=5$, whence $\mathcal{V}(\beta)=V:=\left\{\left(x, x^{2}\right): x^{5}=f_{1}+f_{2} x+f_{3} x^{2}+f_{5} x^{3}+f_{6} x^{4}\right\} ; \quad$ in particular, although $Y^{3} \in \operatorname{lin} . \operatorname{span}\left\{1, X, Y, Y X, Y^{2}\right\}$, the corresponding dependence relation does not cause $\mathcal{V}(\beta)$ to be a proper subset of $V$.

For a numerical example, let $a=1, b=2, c=5, d=14, p=0, q=41$. We find $V=\left\{z_{i}\right\}_{i=1}^{5}$, where $z_{1}=(-1,1), z_{2}=(0,0), z_{3}=(1,1), z_{4}=(-\sqrt{3}, 3), z_{5}=(\sqrt{3}, 3)$, and $\mu_{0}=\frac{1}{4} \delta_{z_{1}}+\frac{1}{3} \delta_{z_{2}}+\frac{1}{4} \delta_{z_{3}}+$ $\frac{1}{12} \delta_{z_{4}}+\frac{1}{12} \delta_{z_{5}}$. For an example illustrating Case I, note that $\mathcal{M}(3)$ (as just described) is flat, and thus admits a unique flat extension $\mathcal{M}(4)$. In $\mathcal{S}_{4}(4)$, the first dependence relation occurs at $Y^{k} X$ with $2=k<n-1=3$, so $\mathcal{M}(4)$ illustrates Case I.

\section{An Application to the Full Moment Problem}

We conclude with the proof of Theorem 2.3: the sequence $\beta \equiv \beta^{(\infty)}$ has a representing measure supported in $y=x^{2}$ if and only if $\mathcal{M}(\infty) \geq 0$ and $Y=X^{2}$ in $\mathcal{C}_{\mathcal{M}(\infty)}$.

The following lemma was given in [Fia3] in terms of the complex moment matrix $M(\infty)(\gamma)$, but it also holds for $\mathcal{M}(\infty)(\beta)$.

Lemma 5.1. ([Fia3, Proposition 4.2]) Let $\beta \equiv \beta^{(\infty)}$ be a full sequence such that $\mathcal{M}(\infty) \geq 0$. Then $\mathcal{M}(n)$ is positive and recursively generated for each $n \geq 1$.

The following is equivalent to a special case for $K \subseteq \mathbb{C}$ of a result proved by J. Stochel for $K \subseteq \mathbb{C}^{n}$ $(n>1)$ [Sto2, Theorem 4].

Theorem 5.2. ([Sto2]) Let $K$ be a closed subset of $\mathbb{R}^{2}$. A full sequence $\beta \equiv \beta^{(\infty)}$ has a representing measure supported in $K$ if and only if, for each $n \geq 1, \beta^{(2 n)}$ has a representing measure supported in $K$.

Proof of Theorem 2.3. The necessity of the conditions is clear. For sufficiency, assume $\mathcal{M}(\infty) \geq 0$ and $Y=X^{2}$. By Lemma 5.1, $\mathcal{M}(n+1)$ is positive and recursively generated for each $n \geq 1$, so $\mathcal{M}(n)$ admits a positive, recursively generated extension, and $Y=X^{2}$ in $\mathcal{C}_{\mathcal{M}(n)}$. By $(i v) \Rightarrow(i i i)$ in Theorem $2.2, \beta^{(2 n)}$ admits a $\operatorname{rank} \mathcal{M}(n)$-atomic measure, necessarily supported in the parabola $y=x^{2}$. By Theorem 5.2, $\beta$ admits a representing measure supported in $y=x^{2}$.

The full moment problem on compact semi-algebraic sets in $\mathbb{R}^{n}$ was solved by K. Schmüdgen [Sch]. Recently, the analysis of the semi-algebraic case was extended to non-compact sets by V. Powers and C. Scheiderer [PoSc] (cf. [KuMa]). The results of [PoSc] include Stochel's parabola theorem (Theorem 2.3) as a special case.

\section{REFERENCES}

[Atz] A. Atzmon, A moment problem for positive measures on the unit disc, Pacific J. Math. 59(1975), 317-325.

[CuFi1] R. Curto and L. Fialkow, Solution of the truncated complex moment problem with flat data, Memoirs Amer. Math. Soc. no. 568, Amer. Math. Soc., Providence, 1996. 
[CuFi2] R. Curto and L. Fialkow, Flat extensions of positive moment matrices: Relations in analytic or conjugate terms, Operator Th.: Adv. Appl. 104(1998), 59-82.

[CuFi3] R. Curto and L. Fialkow, Flat extensions of positive moment matrices: Recursively generated relations, Memoirs Amer. Math. Soc. no. 648, Amer. Math. Soc., Providence, 1998.

[CuFi4] R. Curto and L. Fialkow, The truncated complex K-moment problem, Trans. Amer. Math. Soc. 352(2000), 2825-2855.

[CuFi5] R. Curto ad L. Fialkow, The quadratic moment problem for the unit disk and unit circle, Integral Equations Operator Theory 38(2000), 377-409.

[CuFi6] R. Curto and L. Fialkow, Solution of the singular quartic moment problem, J. Operator Theory 48(2002), 315-354.

[CuFi7] R. Curto and L. Fialkow, A duality proof of Tchakaloff's theorem, J. Math. Anal. Appl. 269(2002), 519-532.

[CuFi8] R. Curto and L. Fialkow, Solution of the truncated hyperbolic moment problem, manuscript in preparation.

[Dou] R.G. Douglas, On majorization, factorization, and range inclusion of operators on Hilbert spaces, Proc. Amer. Math. Soc. 17(1966), 413-415.

[Fia1] L. Fialkow, Positivity, extensions and the truncated complex moment problem, Contemporary Math. 185(1995), 133-150.

[Fia2] L. Fialkow, Minimal representing measures arising from rank-increasing moment matrix extensions, J. Operator Theory 42(1999), 425-436.

[Fia3] L. Fialkow, Truncated complex moment problems with a $Z \bar{Z}$ relation, Integral Equations Operator Theory 45(2003), 405-435.

[KuMa] S. Kuhlmann and M. Marshall, Positivity, sums of squares and the multidimensional moment problem, Trans. Amer. Math.Soc. 354(2002), 4285-4301.

[PoSc] V. Powers and C. Scheiderer, The moment problem for non-compact semialgebraic sets, Advances in Geometry 1(2001), 71-88.

[Put1] M. Putinar, A two-dimensional moment problem, J. Funct. Anal. 80(1988), 1-8.

[Put2] M. Putinar, Positive polynomials on compact semi-algebraic sets, Indiana Univ. Math. J. 42(1993), 969-984.

[PuVa] M. Putinar and F.-H. Vasilescu, Solving moment problems by dimensional extension, Ann. of Math. (2) 149(1999), no. 3, 1087-1107.

[Sch] K. Schmüdgen, The K-moment problem for semi-algebraic sets, Math. Ann. 289(1991), 203-206.

[Smu] J.L. Smul'jan, An operator Hellinger integral (Russian), Mat. Sb. 91(1959), 381-430.

[Sto1] J. Stochel, Moment functions on real algebraic sets, Ark. Mat. 30(1992), 133-148.

[Sto2] J. Stochel, Solving the truncated moment problem solves the moment problem, Glasgow J. Math. 43(2001), 335-341.

[StSz1] J. Stochel and F.H. Szafraniec, Algebraic operators and moments on algebraic sets, Portugal. Math. 51(1994), $25-45$.

[StSz2] J. Stochel and F.H. Szafraniec, The complex moment problem and subnormality: A polar decomposition approach, J. Funct. Anal. 159(1998), 432-491.

[Wol] Wolfram Research, Inc., Mathematica, Version 3.0, Wolfram Research, Inc., Champaign, IL, 1996.

Department of Mathematics, The University of Iowa, Iowa City, Iowa 52242

E-mail address: rcurto@math.uiowa.edu

URL: http://www.math.uiowa.edu/ rcurto/

Department of Computer Science, State University of New York, New Paltz, Ny 12561

E-mail address: fialkowl@newpaltz.edu

$U R L:$ http://www.mcs.newpaltz.edu/faculty/fialkow.html 